\title{
Resultant entropy/information, phase/entropy continuity and bond multiplicities in degenerate electronic states
}

\author{
Roman F. Nalewajski
}

Received: 28 October 2014 / Accepted: 22 December 2014 / Published online: 25 January 2015

C The Author(s) 2015. This article is published with open access at Springerlink.com

\begin{abstract}
The probability and phase/current facets of electronic states generate the classical and nonclassical information terms, respectively. The current-related supplements of the classical information measures and continuity equations for these degrees-of-freedom are summarized. The continuity of the resultant quantum entropy is also explored. This thermodynamic-like description is applied to discuss the temporal aspect of the promolecule-to-molecule transition in $\mathrm{H}_{2}^{+}$. The Wiberg-type bond multiplicity concept is extended to cover the degenerate electronic states. They generally exhibit finite spatial phases and hence nonvanishing electronic currents, and thus also nonzero nonclassical contributions to the resultant content of the state entropy/information. Illustrative example of the excited configurations in the $\pi$ electron ring of benzene is investigated using the complex framework of the (groundstate equivalent) molecular orbitals in Hückel approximation. To validate these generalized concepts, correlations between the $\pi$-bond orders/multiplicities and orbital excitation energies are explored.
\end{abstract}

Keywords Bond multiplicities - Continuity equantions - Electronic phases/currents Excited configurations in benzene $\cdot$ Nonclassical entropy/information · Promoleculeto-molecule transition

\section{Introduction}

The overall entropy/information content of quantum electronic states is one of the crucial problems of the molecular electronic structure. In the past the classical

\footnotetext{
R. F. Nalewajski $(\varangle)$

Department of Theoretical Chemistry, Jagiellonian University,

R. Ingardena 3, 30-060 Cracow, Poland

e-mail: nalewajs@chemia.uj.edu.pl
} 
(probability-based) Information Theory (IT) [1-8] has been successfully applied to explore molecular electron distributions and extract patterns of the system chemical bonds, e.g., [9-18]. It has been recently argued, however, that both the electron probability distribution, determined by the wave-function modulus, and the particle current, related to the gradient of the wave-function phase, ultimately contribute to the resultant information content of molecular states [9,10,19-31]. The particle density reveals only the classical part of the information content, while the wave-function phase or the probability current generate its nonclassical complement in the associated resultant information descriptor. The extremum principles of these generalized information measures determine the quantum equilibria in molecules and their fragments, described by the phase-modified wave functions. The phase/current extension of the ordinary (probability) communication systems has also been introduced [26].

The phenomenological description of molecular equilibria has been proposed, which resembles that developed in ordinary irreversible thermodynamics [32]. These two fundamental degrees of freedom of general, complex molecular states, e.g., the degenerate electronic configurations in molecular orbital (MO) theories, also affect the bond-order and/or chemical multiplicity concepts [33-44]. The latter have been originally formulated for real electronic states approximated by the wave functions of self-consistent field (SCF) theories, from typical SCF LCAO MO calculations using linear combinations (LC) of (real) atomic basis functions, e.g., the atomic orbitals (AO) themselves. These descriptors have been demonstrated to closely follow the intuitive expectations and constitute valuable tools for interpreting molecular wave functions in chemical terms.

We begin the present analysis with a brief summary of the probability and phase degrees-of-freedom in molecular states and their information contributions in the complex electronic states. The combined treatment of the density and phase/current facets of electronic states in a "thermodynamic"-like fashion is advocated. The relevant continuity equations are derived, establishing the current and source concepts of the state phase and its resultant entropy. This phenomenological description is then applied to investigate the relaxation time of the promolecule $\rightarrow$ molecule reorganization in a prototype "half"-bond system of $\mathrm{H}_{2}^{+}$. The bond multiplicity concept is extended into degenerate electronic states, which can generate the complex Charge and Bond-Order (CBO) matrix of the SCF LCAO MO theory, i.e., the one-electron density matrix in $\mathrm{AO}$ representation. These generalized concepts are used to probe the $\pi$-bond pattern in selected excited configurations of the carbon ring in benzene, using the familiar approximation of the Hückel MO theory. We shall also examine how these new concepts correlate with the configuration orbital excitation energy.

Throughout the article the following tensor notation is used: A denotes a scalar, $\boldsymbol{A}$ stands for the row- or column-vector, and $\mathbf{A}$ represents a square or rectangular matrix. The logarithm of the Shannon-type information measure is taken to an arbitrary but fixed base. In keeping with the custom in works on IT the logarithm taken to base $2, \log =\log _{2}$, corresponds to the information measured in bits (binary digits), while selecting $\log =\ln$ expresses the amount of information in nats (natural units): 1 nat $=1.44$ bits . 


\section{Probability and phase/current degrees-of-freedom of electronic states}

For reasons of simplicity, let us first consider a single electron $(N=1)$ in state $|\varphi\rangle \equiv|\psi(0)\rangle$ at time $t=0$, described by the wave function

$$
\varphi(\boldsymbol{r})=\langle\boldsymbol{r} \mid \varphi\rangle=R(\boldsymbol{r}) \exp [\mathrm{i} \phi(\boldsymbol{r})] \equiv \psi(\boldsymbol{r}, t=0),
$$

where $R(\boldsymbol{r})$ and $\phi(\boldsymbol{r})$ stand for its modulus and phase parts, respectively. In what follows we adopt the positive-phase convention: $\phi(\boldsymbol{r})=|\phi(\boldsymbol{r})| \geq 0$. The particle spatial distribution is then described by the electron density $\rho(\boldsymbol{r})=N p(\boldsymbol{r})$ or its probability (shape) factor $p(\boldsymbol{r})$ generated by the square of its classical amplitude $R(\boldsymbol{r})$ :

$$
p(\boldsymbol{r})=\varphi(\boldsymbol{r}) \varphi^{*}(\boldsymbol{r})=R(\boldsymbol{r})^{2} .
$$

In the molecular scenario one envisages this single electron moving in the external potential $v(\boldsymbol{r})=-\sum_{\alpha} Z_{\alpha} /\left|\boldsymbol{r}-\boldsymbol{R}_{\alpha}\right|$ due to the "frozen" nuclei (Born-Oppenheimer approximation). Its dynamics is described by the Hamiltonian

$$
\hat{\mathrm{H}}(\boldsymbol{r})=-\left(\hbar^{2} / 2 m\right) \Delta+v(\boldsymbol{r})=\hat{\mathrm{T}}(\boldsymbol{r})+v(\boldsymbol{r}),
$$

where $\hat{\mathrm{T}}(\boldsymbol{r})=\hat{\mathbf{p}}^{2}(\boldsymbol{r}) / 2 m=-\left(\hbar^{2} / 2 m\right) \Delta$ denotes the kinetic energy operator with the momentum operator $\hat{\mathbf{p}}(\boldsymbol{r})=-\mathrm{i} \hbar \nabla$.

The probability density of Eq. (2) and the gradient of the state phase $\phi(\boldsymbol{r})$ together determine the associated current density,

$$
\begin{aligned}
\boldsymbol{j}(\boldsymbol{r}) & =\langle\varphi|\hat{\mathbf{j}}(\boldsymbol{r})| \varphi\rangle=\frac{\hbar}{2 m i}\left[\varphi^{*}(\boldsymbol{r}) \nabla \varphi(\boldsymbol{r})-\varphi(\boldsymbol{r}) \nabla \varphi^{*}(\boldsymbol{r})\right]=\frac{\hbar}{m} \operatorname{Im}\left[\varphi^{*}(\boldsymbol{r}) \nabla \varphi(\boldsymbol{r})\right] \\
& =\frac{\hbar}{m} p(\boldsymbol{r}) \nabla \phi(\boldsymbol{r}) \equiv p(\boldsymbol{r}) \boldsymbol{V}(\boldsymbol{r}) \equiv \boldsymbol{j}_{p}(\boldsymbol{r})
\end{aligned}
$$

the expectation value of the current operator

$$
\hat{\mathbf{j}}(\boldsymbol{r})=(2 m)^{-1}\left[\hat{\mathbf{p}}\left(\boldsymbol{r}_{1}\right) \delta\left(\boldsymbol{r}_{1}-\boldsymbol{r}\right)+\delta\left(\boldsymbol{r}_{1}-\boldsymbol{r}\right) \hat{\mathbf{p}}\left(\boldsymbol{r}_{1}\right)\right] .
$$

The current-per-particle distribution,

$$
\boldsymbol{j}(\boldsymbol{r}) / p(\boldsymbol{r})=\frac{\hbar}{m} \nabla \phi(\boldsymbol{r}) \equiv \boldsymbol{V}(\boldsymbol{r}),
$$

measures the velocity field $\boldsymbol{V}(\boldsymbol{r})$ of this probability "fluid", which is seen to be determined solely by the gradient of the state phase.

The wave-function modulus $R$, the classical amplitude of the particle probabilitydensity function $p, R=(p)^{1 / 2}$, and the state phase $\phi$, or its gradient $\nabla \phi$ determining the current density $\boldsymbol{j}$, thus constitute two fundamental degrees-of-freedom in the quantum treatment of the electronic states of this one-electron system: $\psi \Leftrightarrow(R, \phi) \Leftrightarrow$ $(p, \boldsymbol{j})$. The eigensolutions of $\hat{\mathrm{H}}(\boldsymbol{r})$ represent the stationary electronic states $\left\{\varphi_{i}(\boldsymbol{r})\right\}$ : 


$$
\hat{\mathrm{H}}(\boldsymbol{r}) \varphi_{i}(\boldsymbol{r})=E_{i} \varphi_{i}(\boldsymbol{r}), \quad i=0,1, \ldots,
$$

which correspond to the sharply specified electronic energies $\left\{E_{i}\right\}$, with the lowest $(i=0)$ eigenvalue marking the energy of the system ground state and the timeindependent probability distribution, $p_{i}(\boldsymbol{r})=\left|\varphi_{i}(\boldsymbol{r}, t)\right|^{2}=\left|\varphi_{i}(\boldsymbol{r})\right|^{2}$, which identifies the system "weak"-stationary character. Typically $\varphi_{i}(\boldsymbol{r}, t)$ also exhibits the purely timedependent phase $\phi_{i}(t)$, i.e., the exactly vanishing spatial-phase component $\phi_{i}(\boldsymbol{r})=0$, in the particle full (complex) wave function

$$
\begin{aligned}
\psi_{i}(\boldsymbol{r}, t) & =\left\langle\boldsymbol{r} \mid \psi_{i}(t)\right\rangle=R_{i}(\boldsymbol{r}) \exp \left\{\mathrm{i}\left[\phi_{i}(\boldsymbol{r})+\phi_{i}(t)\right]\right. \\
& =\varphi_{i}(\boldsymbol{r}) \exp \left[-\mathrm{i}\left(E_{i} / \hbar\right) t\right] \equiv R_{i}(\boldsymbol{r}) \exp \left[-\mathrm{i} \omega_{i} t\right],
\end{aligned}
$$

and hence both the stationary probability distribution, $p_{i}(\boldsymbol{r}, t)=p_{i}(\boldsymbol{r})=\left|\varphi_{i}(\boldsymbol{r})\right|^{2}=$ $R_{i}(\boldsymbol{r})^{2}$, and the vanishing current density $\boldsymbol{j}_{i}(\boldsymbol{r})=(\hbar / m) p_{i}(\boldsymbol{r}) \nabla \phi_{i}(\boldsymbol{r})=\boldsymbol{0}$ marking the "strong"-stationary state. In particular, the exact (non-degenerate) ground state indeed corresponds to the vanishing spatial phase, $\phi_{0}(\boldsymbol{r})=0$, in its general (complex) form $\varphi_{0}(\boldsymbol{r})=R_{0}(\boldsymbol{r}) \exp \left[\mathrm{i} \phi_{0}(\boldsymbol{r})\right]=R_{0}(\boldsymbol{r})$, and hence the vanishing current distribution $\boldsymbol{j}_{0}(\boldsymbol{r})=\boldsymbol{0}$, thus fulfilling both the necessary ("weak") and sufficient ("strong") conditions of the state true stationarity.

However, the familiar examples of degenerate electronic states in molecules and of the plane-wave state of a free particle show that satisfying the necessary condition, of the system weak stationary character, i.e., of the time-independent probability distribution, does not always imply the fulfillment of the sufficient condition, of the strong stationary state, i.e., of the state vanishing current. Indeed, in the latter case the wave function $\varphi_{k}(\boldsymbol{r}, t)=A \exp \left[\mathrm{i}\left(\boldsymbol{k} \cdot \boldsymbol{r}-\omega_{k} t\right)\right]$ describes only the "weak"-stationary state, $p_{\boldsymbol{k}}(\boldsymbol{r}, t)=p_{\boldsymbol{k}}=|A|^{2}$, and generates the finite local current $\boldsymbol{j}_{\boldsymbol{k}}(\boldsymbol{r})=\boldsymbol{j}_{\boldsymbol{k}}=(\hbar / m) p_{\boldsymbol{k}} \boldsymbol{k}=|A|^{2} \boldsymbol{V}$, where the particle classical velocity (momentum $\boldsymbol{p}_{\boldsymbol{k}}$ per unit mass) $\boldsymbol{V}=\boldsymbol{p}_{\boldsymbol{k}} / \mathrm{m}$.

This one-electron development can be straightforwardly generalized into the $N$ electronic states in the familiar orbital approximation, described by Slater determinants of the occupied single-electron states [9,10,19-31]. Since the electronic density, current, and entropy or information operators are one-electron in character, the expectation values of their sums over all constituent electrons are given by the sums of their expectation values in each occupied MO. In constructing the Slater determinant reproducing the specified electron density one uses the Harriman-Zumbach-Maschke (HZM) construction [45,46] of the Density Functional Theory (DFT) [47-49], which realizes the crucial insights due to Macke [50] and Gilbert [51].

\section{Classical entropy/information measures and their nonclassical supplements}

Let us briefly summarize the entropy/information concepts of classical IT [1-8]. The historically first local measure of Fisher [1,2] provides the average information content in the probability density $p(\boldsymbol{r})$ reminiscent of von Weizsäcker's [52] inhomogeneity correction to the kinetic energy functional in the Thomas-Fermi-Dirac theory, 


$$
I[p]=\int[\nabla p(\boldsymbol{r})]^{2} / p(\boldsymbol{r}) d \boldsymbol{r} \equiv \int p(\boldsymbol{r}) I_{p}(\boldsymbol{r}) d \boldsymbol{r},
$$

where $I_{p}(\boldsymbol{r})=[\nabla p(\boldsymbol{r}) / p(\boldsymbol{r})]^{2} \equiv I^{\text {class. }}(\boldsymbol{r})$ stands for the associated information density-per-electron. This probability functional is simplified when expressed in terms of its classical probability aplitude $R(\boldsymbol{r})=\sqrt{p(\boldsymbol{r})}$,

$$
\begin{aligned}
I[p] & =4 \int[\nabla R(\boldsymbol{r})]^{2} d \boldsymbol{r} \equiv I[R] \equiv I^{\text {class. }}[\varphi]=\left\langle\varphi\left|\hat{\mathrm{I}}_{p}\right| \varphi\right\rangle, \\
\hat{\mathrm{I}}_{p}(\boldsymbol{r}) & =I_{p}(\boldsymbol{r})
\end{aligned}
$$

thus revealing that it effectively measures the average length of the gradient of the state modulus function $R(\boldsymbol{r})$.

The global measure of Shannon $[3,4]$ provides a complementary to $I[p]$ classical descriptor of the average information in $p(\boldsymbol{r})$, called the Shannon entropy,

$$
\begin{aligned}
S[p] & =-\int p(\boldsymbol{r}) \log p(\boldsymbol{r}) d \boldsymbol{r} \equiv \int p(\boldsymbol{r}) S_{p}(\boldsymbol{r}) d \boldsymbol{r} \equiv S^{\text {class. }}[\varphi]=\left\langle\varphi\left|\hat{\mathbf{S}}_{p}\right| \varphi\right\rangle, \\
\hat{\mathrm{S}}_{p}(\boldsymbol{r}) & =-\log p(\boldsymbol{r})=S_{p}(\boldsymbol{r}),
\end{aligned}
$$

where $S_{p}(\boldsymbol{r}) \equiv S^{\text {class. }}(\boldsymbol{r})$ measures the density-per-electron of this classical descriptor. The Fisher information $I[p]$ measures the distribution "narrowness" (determinicity, order), while the complementary Shannon descriptor $S[p]$ reflects the probability "spread" (indeterminicity, disorder). Thus, the less Shannon entropy in the probability distribution, of the indeterminicity information, implies the more electronic gradient information, of Fisher's determinicity descriptor. This "inverse" character of these classical measures also extends into their resultant analogs including the nonclassical entropy/information components [21,28].

A presence of a finite spatial phase $\phi(\boldsymbol{r})$, i.e., of a nonvanishing electronic current related to the phase gradient, signifies a displacement from the system ("strong") stationarity; the less stringent ("weak") stationarity requirement, of the time-independent probability distribution, admits a nonvanishing spatial phase of the system wave function generating electronic current. This is the case in the molecular equilibrium states [9,10,19-31], which maximize the state resultant entropy. The presence of a finite current introduces the additional "structure" element of quantum systems, affecting the system resultant entropy/information. This finite current pattern implies less "uncertainty" (more "order") in the molecular electronic state compared to its classical information content, i.e., the negative sign of the nonclassical entropy supplement. Therefore, the complex quantum states should be expected to exhibit less resultant indeterminicity information (more resultant determinicity information), compared to the stationary states of the same probability distribution.

The resultant Fisher information combines the classical kinetic-energy contribution, from the gradient of the state modulus, and the nonclassical kinetic-energy term due tho the phase gradient. This implies a positive sign of the nonclassical phase contribution to resultant gradient determinicity information. For a single electron in state $\varphi(\boldsymbol{r})$ the resultant gradient measure of the information content, related to the system average electronic kinetic energy, 


$$
T[\varphi]=\langle\varphi|\hat{\mathrm{T}}| \varphi\rangle=-\frac{\hbar^{2}}{2 m} \int \varphi^{*}(\boldsymbol{r}) \Delta \varphi(\boldsymbol{r}) d \boldsymbol{r}=\frac{\hbar^{2}}{2 m} \int|\nabla \varphi(\boldsymbol{r})|^{2} d \boldsymbol{r} .
$$

contains both the classical (probability) and nonclassical (phase/current) components:

$$
\begin{aligned}
I[\varphi] & =\langle\varphi|\hat{\mathrm{I}}| \varphi\rangle=4 \int|\nabla \varphi(\boldsymbol{r})|^{2} d \boldsymbol{r}=\left(8 m / \hbar^{2}\right) T[\varphi] \\
& =I[p]+4 \int p(\boldsymbol{r})[\nabla \phi(\boldsymbol{r})]^{2} d \boldsymbol{r} \equiv I[p]+I[p, \phi] \\
& =I[p]+\left(\frac{2 m}{\hbar}\right)^{2} \int j^{2}(\boldsymbol{r}) / p(\boldsymbol{r}) d \boldsymbol{r} \equiv I[p]+I[p, \boldsymbol{j}] \\
& \equiv I^{\text {class. }}[\varphi]+I^{\text {nclass. }}[\varphi] \equiv \int p(\boldsymbol{r})\left[I_{p}(\boldsymbol{r})+I_{\phi}(\boldsymbol{r})\right] d \boldsymbol{r} \equiv \int p(\boldsymbol{r}) I(\boldsymbol{r}) d \boldsymbol{r} .
\end{aligned}
$$

This overall quantum Fisher information is seen to probe the length of the gradient $\nabla \varphi(\boldsymbol{r})$ of the state quantum amplitude (wave function). This generalized gradient determinicity information for locality events combines both the classical, von Weizsäcker's functional $I^{\text {class. }}[\varphi]=I[p]=\int p(\boldsymbol{r}) I_{p}(\boldsymbol{r}) d \boldsymbol{r}$, and the nonclassical term $I^{\text {nclass. }}[\varphi]=\int p(\boldsymbol{r}) I_{\phi}(\boldsymbol{r}) d \boldsymbol{r}=I[p, \phi]=I[p, \boldsymbol{j}]$ due to the state phase (or probability current), reflecting the square of the phase gradient. The relevant information densities-per-electron,

$$
\begin{aligned}
I^{\text {class. }}(\boldsymbol{r}) & =4[\nabla R(\boldsymbol{r})]^{2} \equiv I_{p}(\boldsymbol{r}) \text { and } \\
I^{\text {nclass. }}(\boldsymbol{r}) & =4[\nabla \phi(\boldsymbol{r})]^{2}=(2 m / \hbar)^{2}[\boldsymbol{j}(\boldsymbol{r}) / p(\boldsymbol{r})]^{2} \equiv I_{\phi}(\boldsymbol{r}),
\end{aligned}
$$

define the (multiplicative) information operator of Eq. (12): $\hat{\mathrm{I}}(\boldsymbol{r})=I_{p}(\boldsymbol{r})+I_{\phi}(\boldsymbol{r})=$ $I(\boldsymbol{r})$.

One also observes that the densities-per-electron of the classical entropy and gradient information are mutually related $[9,10]$ :

$$
I^{\text {class. }}(\boldsymbol{r})=[\nabla \ln p(\boldsymbol{r})]^{2}=\left[\nabla S^{\text {class. }}(\boldsymbol{r})\right]^{2} .
$$

Thus, the square of the gradient of the Shannon probe of the state indeterminicity information generates the density of the associated Fisher measure of the state determinicity information.

The nonclassical complement of the Shannon entropy, $S^{\text {nclass. }}[\varphi][9,19,22-27]$,

$$
\begin{aligned}
S^{\text {nclass. }}[\varphi] & =\left\langle\varphi\left|\hat{\mathbf{S}}_{\varphi}\right| \varphi\right\rangle=-2 \int p(\boldsymbol{r}) \phi(\boldsymbol{r}) d \boldsymbol{r}=S[p, \phi] \equiv \int p(\boldsymbol{r}) S_{\varphi}(\boldsymbol{r}) d \boldsymbol{r} \leq 0, \\
\hat{\mathbf{S}}_{\varphi}(\boldsymbol{r}) & =-2 \phi(\boldsymbol{r})=S^{\text {nclass. }}(\boldsymbol{r})=S_{\varphi}(\boldsymbol{r}),
\end{aligned}
$$

exhibits a nonpositive density proportional to the local magnitude of the phase function, $|\phi|=\left[\phi^{2}\right]^{1 / 2} \equiv \phi \geq 0$, the square root of the phase-density $\pi=\phi^{2}$, with the particle probability $p$ providing a local "weighting" factor. This functional characterizes a displacement from the strong-stationary equilibrium in terms of the average magnitude 
of the state spatial phase. It complements the classical Shannon entropy of Eq. (10) in the resultant measure of the quantum indeterminicity content in both the probability and current distributions of the complex electronic state $\varphi$ :

$$
\begin{aligned}
& S[\varphi]=S^{\text {class. }}[\varphi]+S^{\text {nclass. }}[\varphi]=\langle\varphi|\hat{\mathrm{S}}| \varphi\rangle \equiv \int p(\boldsymbol{r}) S(\boldsymbol{r}) d \boldsymbol{r}, \\
& \hat{\mathrm{S}}(\boldsymbol{r})=S_{p}(\boldsymbol{r})+S_{\phi}(\boldsymbol{r})=S(\boldsymbol{r})=-\ln p(\boldsymbol{r})-2 \phi(\boldsymbol{r}) .
\end{aligned}
$$

The relation of Eq. (14) then also applies to the nonclassical entropy/information densities [9,10,19-31]:

$$
I^{\text {nclass. }}(\boldsymbol{r})=\left(\frac{2 m \boldsymbol{j}(\boldsymbol{r})}{\hbar p(\boldsymbol{r})}\right)^{2}=4[\nabla \phi(\boldsymbol{r})]^{2} \equiv\left[\nabla S^{\text {nclass. }}(\boldsymbol{r})\right]^{2} .
$$

The nondegenerate ground state $\Psi_{0}$, for which $\phi_{0}(\boldsymbol{r})=0$ and hence $\boldsymbol{j}_{0}(\boldsymbol{r})=\boldsymbol{0}$, corresponds to the so called vertical equilibrium marking the phase-maximum of the nonclassical entropy component, i.e., of the nonclassical indeterminicity-information content, $S^{\text {nclass. }}\left[\phi_{0}\right] \equiv 0$, in close analogy to the maximum-entropy principle of the ordinary thermodynamics [32]. Therefore, any local phase displacement from this strong stationary state, $\delta \phi(\boldsymbol{r})=\phi(\boldsymbol{r})-\phi_{0}(\boldsymbol{r})=\phi(\boldsymbol{r})$, lowers the nonclassical entropy component, $\delta S^{n c l a s s} .[\delta \phi]=S\left[p_{0}, \phi\right] \leq 0$, where $p_{0}(\boldsymbol{r})$ stands for the (constrained) electron probability distribution in the ground-state. The complementary picture emerges, when one adopts the Fisher-type measure of the gradient information content. Now this vertical equilibrium for the fixed ground-state probability distribution $p_{0}(\boldsymbol{r})$ represents the minimum of the nonclassical determinicityinformation content: $I^{\text {nclass. }}\left[\phi_{0}\right] \equiv I\left[p_{0}, \phi_{0}\right]=0$. Thus, any phase displacement from $\phi_{0}$ increases the nonclassical (phase-gradient) information content by $\delta I^{\text {nclass }} \cdot[\delta \phi]=\delta I^{\text {nclass }} \cdot[\phi] \equiv I\left[p_{0}, \phi\right] \geq 0$.

In a search for thermodynamic analogies of interest also is the concept of the gradient measure of the state resultant entropy (quantum indeterminicity-information) $[20,21]$ :

$$
\tilde{I}[\varphi]=I[p]-I[p, \phi] \equiv \tilde{I}^{\text {class. }}[\varphi]+\tilde{I}^{\text {nclass. }}[\varphi] .
$$

Its classical part is determined by the ordinary Fisher information in the probability distribution, $\tilde{I}^{\text {class. }}[\varphi]=I[p]$, while the nonclassical complement is now nonpositive, $\tilde{I}^{\text {nclass. }}[\varphi]=-I[p, \phi] \leq 0$, to conform to the sign of the nonclassical entropy of Eq. (16). Indeed, the classical entropy (average uncertainty) reflects the information received, when the indeterminicity about the electron position in the distribution $p(\boldsymbol{r})$ is removed by an appropriate measurement. This justifies a selection of the classical part of this gradient entropy measure. It has been demonstrated elsewhere [28,29], that both $S[\varphi]$ and $\tilde{I}[\varphi]$ indeed predict the same solutions of the so called horizontalequilibrium problem, of the extremum of the resultant quantum entropy.

To summarize, the system electron distribution, related to the wave-function modulus, reveals the probability (classical) aspect of the molecular information content, while the phase(current) facet of the molecular state gives rise to the specifically quantum (nonclassical) entropy/information terms. Together these two contributions 
allow one to monitor the full (resultant) information content of, say, non-equilibrium or variational quantum states, thus providing the complete information description of their evolution towards the final equilibrium.

The negative sign of the nonclassical global and gradient entropy contributions can be justified by comparing the phase/current entropy in the (one-dimensional) traveling and standing waves of the same amplitude. The strong-stationary distribution of a "standing" wave, resulting from the equal, 50\% probabilities of the "left" and "right" "traveling" waves and hence the vanishing average current, predicts $S[p, \phi]=I[p, \phi]=0$. The weak-stationary distribution of the traveling-wave, say $100 \%$ "right" plane-wave, represents a finite current in this direction thus giving rise to $S[p, \phi]<0$ and $\tilde{I}[p, \phi]<0$. This qualitative result reproduces correctly the intuitive expectation that we have more electronic determinicity (less uncertainty) in the traveling-wave situation, in which the direction of the flux is precisely known, compared to the standing-wave case, in which we are completely ignorant of the current direction.

\section{Phase-continuity}

We continue the representative one-electron development of the preceding section. The quantum dynamics of general electronic states,

$$
\psi(\boldsymbol{r}, t) \equiv\langle\boldsymbol{r} \mid \psi(t)\rangle=R(\boldsymbol{r}, t) \exp [\mathrm{i} \phi(\boldsymbol{r}, t)]
$$

is determined by the Schrödinger equation (SE):

$$
(\mathrm{i} \hbar)^{-1} \hat{\mathrm{H}} \psi=(\partial \psi / \partial t) .
$$

This equation and its complex conjugate,

$$
-(\mathrm{i} \hbar)^{-1} \hat{\mathrm{H}} \psi^{*}=\left(\partial \psi^{*} / \partial t\right)
$$

can be subsequently used to determine the time evolution of the probability distribution $p(\boldsymbol{r}, t)$ or its classical amplitude $R(\boldsymbol{r}, t)$, and to establish the associated dynamics of the state phase $\phi(\boldsymbol{r}, t)$ or its square, the phase density $\pi(\boldsymbol{r}, t)=\phi(\boldsymbol{r}, t)^{2}$.

Let us multiply Eq. (20a) by $\psi^{*}$ and Eq. (20b) by $\psi$ :

$$
\begin{aligned}
(\mathrm{i} \hbar)^{-1} \psi^{*} \hat{\mathrm{H}} \psi & =(\mathrm{i} \hbar)^{-1}\left(\psi^{*} \hat{\mathrm{T}} \psi+p v\right) \\
& =-[\hbar /(2 m \mathrm{i})] \psi^{*} \Delta \psi-\mathrm{i}(p / \hbar) v=\psi^{*}(\partial \psi / \partial t) \text { and } \\
-(\mathrm{i} \hbar)^{-1} \psi \hat{\mathrm{H}} \psi^{*} & =-(\mathrm{i} \hbar)^{-1}\left(\psi \hat{\mathrm{T}} \psi^{*}+p v\right) \\
& =[\hbar /(2 m \mathrm{i})] \psi \Delta \psi^{*}+\mathrm{i}(p / \hbar) v=\psi\left(\partial \psi^{*} / \partial t\right) .
\end{aligned}
$$

Taking first the sum of these two equations gives the familiar continuity equation for the electronic probability density:

$$
(\mathrm{i} \hbar)^{-1}\left(\psi^{*} \hat{\mathrm{T}} \psi-\psi \hat{\mathrm{T}} \psi^{*}\right)=-[\hbar /(2 m \mathrm{i})]\left(\psi^{*} \Delta \psi-\psi \Delta \psi^{*}\right)=-\nabla \cdot \boldsymbol{j}=\partial p / \partial t .
$$


This (sourceless) balance relation of the electronic distribution,

$$
d p / d t \equiv \dot{p}=\sigma_{p}=\partial p / \partial t+\nabla \cdot \boldsymbol{j}=0
$$

also implies the conservation in time of the probability (wave function) normalization:

$$
d\langle\psi(t) \mid \psi(t)\rangle / d t=d\left[\int p(\boldsymbol{r}, t) d \boldsymbol{r}\right] / d t=\int[d p(\boldsymbol{r}, t) / d t] d \boldsymbol{r}=0 .
$$

The difference of Eqs. (21) similarly generates the time-derivative of the state phase $\phi(\boldsymbol{r}, t)$ :

$$
\begin{aligned}
(\mathrm{i} \hbar)^{-1}\left(\psi^{*} \hat{\mathrm{T}} \psi+\psi \hat{\mathrm{T}} \psi^{*}+2 p v\right) & =-[\hbar /(2 m \mathrm{i})]\left(\psi^{*} \Delta \psi+\psi \Delta \psi^{*}\right)+(\mathrm{i} \hbar)^{-1} 2 p v \\
& =-[\hbar /(m \mathrm{i})]\left[R \Delta R-p(\nabla \phi)^{2}\right]+(\mathrm{i} \hbar)^{-1} 2 p v \\
& =\psi^{*}(\partial \psi / \partial t)-\psi\left(\partial \psi^{*} / \partial t\right)=2 \mathrm{i} p(\partial \phi / \partial t) \text { or } \\
(\partial \phi / \partial t) & =[\hbar /(2 m)]\left[R^{-1} \Delta R-(\nabla \phi)^{2}\right]-v / \hbar .
\end{aligned}
$$

This derivative also implies the associated dynamics of the phase density $\pi(\boldsymbol{r}, t)=$ $\phi(\boldsymbol{r}, t)^{2}$ :

$$
\partial \pi / \partial t=2 \phi(\partial \phi / \partial t)=(\hbar \phi / m)\left[R^{-1} \Delta R-(\nabla \phi)^{2}\right]-2 \phi v / \hbar .
$$

Therefore, in general molecular states of Eq. (19) the wave-function phase is evolving in time thus changing the phase-related contributions to the resultant descriptors of the information content. The time rate of change in the state phase is seen to be determined by spatial variations of both the modulus and phase components of molecular electronic states, as well as by the shape of the external potential.

Ascribing the flux concept to the phase aspect of molecular states, which ultimately determines the partition of this time-derivative between the "outflow" and "source" contributions in the underlying phase-continuity equation, is not unique. In the past several alternative choices of the phase-current have been proposed, differing in their implicit definitions of the representative "velocity" $V_{\pi}(\boldsymbol{r})$ of the phase "fluid":

$$
\pi(\boldsymbol{r}, t)=\pi\left[\boldsymbol{r}-\boldsymbol{V}_{\pi}(\boldsymbol{r}) t\right] \text { or } \pi\left(\boldsymbol{r}+\boldsymbol{V}_{\pi}(\boldsymbol{r}) \Delta t, t+\Delta t\right)=\pi(\boldsymbol{r}, t) .
$$

Different phase-flow concepts only reshuffle the time rate of change in the state phasedensity between the outflow and source contributions in the underlying phase continuity equation.

It should be observed that in quantum mechanics the particle speed,

$$
\langle\varphi|\hat{\mathbf{V}}(\boldsymbol{r})| \varphi\rangle=p(\boldsymbol{r})^{-1}\langle\varphi|\hat{\mathbf{j}}(\boldsymbol{r})| \varphi\rangle=\boldsymbol{V}(\boldsymbol{r}),
$$

the expectation value of the (Hermitian) velocity operator [Eq. (5)],

$$
\hat{\mathbf{V}}(\boldsymbol{r})=[2 m p(\boldsymbol{r})]^{-1}\left[\hat{\mathbf{p}}(\boldsymbol{r}) \delta\left(\boldsymbol{r}_{1}-\boldsymbol{r}\right)+\delta\left(\boldsymbol{r}_{1}-\boldsymbol{r}\right) \hat{\mathbf{p}}(\boldsymbol{r})\right],
$$


is not sharply specified simultaneously with the given electron position $\boldsymbol{r}$. Although such a phase-velocity in molecular quantum mechanics generally differs from the classical speed $\boldsymbol{V}_{\text {class }}=\boldsymbol{p} / m=\boldsymbol{V}$ of the free particle exhibiting the momentum $\boldsymbol{p}=\hbar \boldsymbol{k}$, the most natural choice seems to follow from the requirement that the phase "current" is indeed effected by the movement of the probability fluid itself [see Eq. (5)]: $\boldsymbol{V}_{\pi}(\boldsymbol{r})=\boldsymbol{V}(\boldsymbol{r})$. In this perspective the flow of the particle probability is also responsible for the flow of the state phase. In other words, it is the electrons which also carry the density of the phase in the molecular state. Such a definition of the phase velocity gives rise to the flow descriptor

$$
\boldsymbol{J}(\boldsymbol{r}, t)=\pi(\boldsymbol{r}, t) \boldsymbol{V}(\boldsymbol{r}, t)=\frac{\hbar}{m} \pi(\boldsymbol{r}, t) \nabla \phi(\boldsymbol{r}, t) \equiv \boldsymbol{J}_{\pi}(\boldsymbol{r}, t)
$$

related to the probability current of Eq. (4a):

$$
\boldsymbol{J}(\boldsymbol{r})=[\pi(\boldsymbol{r}) / p(\boldsymbol{r})] \boldsymbol{j}(\boldsymbol{r}) .
$$

The corresponding (purely $\phi$-dependent) divergence term,

$$
\begin{aligned}
\nabla \cdot \boldsymbol{J}(\boldsymbol{r}) & =\frac{\hbar}{m}[\nabla \pi(\boldsymbol{r}) \cdot \nabla \phi(\boldsymbol{r})+\pi(\boldsymbol{r}) \Delta \phi(\boldsymbol{r})]=\frac{\hbar \phi(\boldsymbol{r})}{m}\left\{2[\nabla \phi(\boldsymbol{r})]^{2}+\phi(\boldsymbol{r}) \Delta \phi(\boldsymbol{r})\right\} \\
& =\nabla[\pi(\boldsymbol{r}) / p(\boldsymbol{r})] \cdot \boldsymbol{j}(\boldsymbol{r})+[\pi(\boldsymbol{r}) / p(\boldsymbol{r})] \nabla \cdot \boldsymbol{j}(\boldsymbol{r}),
\end{aligned}
$$

then determines the outflow part in the associated phase-continuity equation,

$$
\partial \pi(\boldsymbol{r}) / \partial t=-\nabla \cdot \boldsymbol{J}(\boldsymbol{r})+\sigma_{\pi}(\boldsymbol{r}),
$$

thus also identifying the associated phase-source contribution:

$$
\begin{aligned}
\sigma_{\pi}(\boldsymbol{r}) & =d \pi(\boldsymbol{r}) / d t=\partial \pi(\boldsymbol{r}) / \partial t+\nabla \cdot \boldsymbol{J}(\boldsymbol{r}) \\
& =\phi(\boldsymbol{r})\left\{(\hbar / m)\left[R(\boldsymbol{r})^{-1} \Delta R(\boldsymbol{r})+\phi(\boldsymbol{r}) \Delta \phi(\boldsymbol{r})+(\nabla \phi(\boldsymbol{r}))^{2}\right]-2 v(\boldsymbol{r}) / \hbar\right\} \neq 0 .
\end{aligned}
$$

The latter is seen to identically vanish only for real wave functions, when $\phi(\boldsymbol{r})=0$, e.g., in the strong-stationary state of Eq. (6).

Another phase-current concept follows from the principle of the maximum symmetry in treating the modulus and phase degrees-of-freedom of the molecular electronic states. Since the probability current of Eq. (5) explores the probability density $p(\boldsymbol{r})$ and the phase gradient $\nabla \phi(r)$, one assumes, for greater symmetry, that the phase current should similarly explore the phase density $\pi(\boldsymbol{r})$ and the modulus gradient $\nabla R(\boldsymbol{r})$. This produces the most symmetrical definitions of the two flux quantities,

$$
\begin{aligned}
& \boldsymbol{j}(\boldsymbol{r})=\frac{\hbar}{m} p(\boldsymbol{r}) \nabla \phi(\boldsymbol{r})=\frac{\hbar}{2 m} \frac{p(\boldsymbol{r})}{\phi(\boldsymbol{r})} \nabla \pi(\boldsymbol{r}) \text { and } \\
& \boldsymbol{J}(\boldsymbol{r})=\frac{\hbar}{m} \pi(\boldsymbol{r}) \nabla R(\boldsymbol{r})=\frac{\hbar}{2 m} \frac{\pi(\boldsymbol{r})}{R(\boldsymbol{r})} \nabla p(\boldsymbol{r}) \equiv \boldsymbol{J}^{s y m .}(\boldsymbol{r}),
\end{aligned}
$$

related by a variant of Eq. (31): 


$$
\boldsymbol{J}^{s y m} \cdot(\boldsymbol{r})=\left[\frac{\pi(\boldsymbol{r})}{p(\boldsymbol{r})} \frac{\nabla R(\boldsymbol{r})}{\nabla \phi(\boldsymbol{r})}\right] \boldsymbol{j}(\boldsymbol{r}) .
$$

This choice generates the following divergence and source terms, which mix both the $p($ or $R$ ) and $\pi$ (or $\phi)$ dependencies:

$$
\begin{aligned}
\nabla \cdot \boldsymbol{J}^{s y m} \cdot(\boldsymbol{r})= & \frac{\hbar \phi(\boldsymbol{r})}{m}\{2[\nabla \phi(\boldsymbol{r})] \cdot[\nabla R(\boldsymbol{r})]+\phi(\boldsymbol{r}) \Delta R(\boldsymbol{r})\}, \\
\sigma_{\pi}^{s y m \cdot}(\boldsymbol{r})= & \partial \pi(\boldsymbol{r}) / \partial t+\nabla \cdot \boldsymbol{J}^{s y m \cdot}(\boldsymbol{r}) \\
= & \phi(\boldsymbol{r})\left\{( \hbar / m ) \left(\Delta R(\boldsymbol{r})\left[\phi(\boldsymbol{r})+R^{-1}(\boldsymbol{r})\right]\right.\right. \\
& +\nabla \phi(\boldsymbol{r}) \cdot[2 \nabla R(\boldsymbol{r})-\nabla \phi(\boldsymbol{r})])-2 v(\boldsymbol{r}) / \hbar\} .
\end{aligned}
$$

Since different concepts of the phase-flux only redistribute the known time-rate of the phase density [Eq. (26)] between the outflow (divergence) and source contributions in the phase balance (continuity) Eq. (33), one could also, for definiteness, ascribe the whole time derivative of this equation either exclusively to the phase source, for the identically vanishing phase current,

$$
\partial \pi(\boldsymbol{r}) / \partial t \equiv \sigma_{\pi}^{a b s .}(\boldsymbol{r}) \text { and } \quad \boldsymbol{J}_{\pi}(\boldsymbol{r}) \equiv \mathbf{0}
$$

or exclusively to the phase-outflow, for the vanishing source contribution,

$$
\partial \pi(\boldsymbol{r}) / \partial t \equiv-\nabla \cdot \boldsymbol{J}^{a b s} \cdot(\boldsymbol{r}) \text { and } \sigma_{\pi}(\boldsymbol{r})=0 .
$$

Each choice has its interpretative advantages: the former offers the strong-stationary perspective, while the latter provides the sourceless balance outlook [compare Eqs. (22) and (23)] on the time evolution of the phase component of electronic states.

\section{Continuity of resultant entropy}

Consider next a related problem of the source term in the continuity equation for the state resultant entropy $S[\varphi]$ [Eq. (16)], which combines positive classical (probabilityspread) measure of Shannon entropy, $S[p] \equiv S^{\text {class. }}[\varphi]$, and (negative) nonclassical supplement reflecting the average magnitude of the state phase, $S[\pi] \equiv S^{\text {nclass }} \cdot[\varphi]$,

$$
\begin{aligned}
S[\varphi] & =S^{\text {class. }}[\varphi]+S^{\text {nclass. }}[\varphi] \\
& =-\int p(\boldsymbol{r}) \ln p(\boldsymbol{r}) d \boldsymbol{r}-2 \int p(\boldsymbol{r}) \pi(\boldsymbol{r})^{1 / 2} d \boldsymbol{r} \\
& \equiv S[p, \pi] \equiv \int s(\boldsymbol{r}) d \boldsymbol{r} .
\end{aligned}
$$

Following the standard approach of the ordinary thermodynamics [32], we first identify the entropy conjugates of the two independent density variables: $p=|\varphi|^{2}=R^{2}$ and $\pi=\phi^{2}$. For the adopted positive phase convention, $\phi>0$, when $\pi^{1 / 2}=|\phi|=\phi$, one then finds: 


$$
\begin{aligned}
& F_{p}(\boldsymbol{r})=\delta S[\varphi] / \delta p(\boldsymbol{r})=\frac{\partial s(\boldsymbol{r})}{\partial p(\boldsymbol{r})}=-\ln p(\boldsymbol{r})-2 \pi(\boldsymbol{r})^{1 / 2}-1=-\ln p(\boldsymbol{r})-2 \phi(\boldsymbol{r})-1, \\
& F_{\pi}(\boldsymbol{r})=\delta S[\varphi] / \delta \pi(\boldsymbol{r})=\frac{\partial s(\boldsymbol{r})}{\partial \pi(\boldsymbol{r})}=-p(\boldsymbol{r}) / \pi(\boldsymbol{r})^{1 / 2}=-p(\boldsymbol{r}) / \phi(\boldsymbol{r})
\end{aligned}
$$

These entropy-conjugates of the probability and phase densities then determine the associated affinities, "thermodynamic" forces, defined by their gradients:

$$
\begin{aligned}
& \boldsymbol{G}_{p}=\nabla F_{p}=-\left[p^{-1} \nabla p+\pi^{-1 / 2} \nabla \pi\right]=-2\left[R^{-1} \nabla R+\nabla \phi\right] \text { and } \\
& \boldsymbol{G}_{\pi}=\nabla F_{\pi}=\pi^{-1 / 2}\{[p /(2 \pi)] \nabla \pi-\nabla p\}=(R / \phi)[(R / \phi) \nabla \phi-2 \nabla R] .
\end{aligned}
$$

It follows from these definitions that in the strong-stationary state $\varphi_{j}$, when $\phi\left[\varphi_{j}\right]=0$ and $\boldsymbol{j}\left[\varphi_{j}\right]=\boldsymbol{0}, S[\varphi]=S^{\text {class }}[\varphi]$. Therefore, in such states $F_{\pi}=0$ and hence the phase-affinity $\boldsymbol{G}_{\pi}=\nabla F_{\pi}$ identically vanishes, while the probability affinity remains finite. One also observes that in the horizontal equilibrium state $[24,25]$,

$$
\begin{aligned}
& \varphi_{e q .}(\boldsymbol{r})=\varphi(\boldsymbol{r}) \exp \left(\mathrm{i} \phi_{e q .}[p ; \boldsymbol{r}]\right), \\
& \phi_{e q .}[p ; \boldsymbol{r}] \equiv \phi_{e q .}(\boldsymbol{r})=-(1 / 2) \ln p(\boldsymbol{r})=\phi\left[\varphi_{e q .}\right] \geq 0,
\end{aligned}
$$

which marks the phase-extremum of the resultant entropy $S[\varphi]$, the probability affinity vanishes while the phase affinity remains finite.

Let us now reexamine the phase-equilibrium conditions, of the vanishing forces $\boldsymbol{G}_{p}(\boldsymbol{r})$ or $\boldsymbol{G}_{\pi}(\boldsymbol{r})$. The first condition, $\boldsymbol{G}_{p}(\boldsymbol{r})=\boldsymbol{0}$, determines the equilibrium state and phase of the preceding equation, for which the probability current

$$
\boldsymbol{j}\left[\varphi_{e q .}\right] \equiv \boldsymbol{j}_{e q .}=-[\hbar /(2 m)] \nabla p
$$

The second criterion, $\boldsymbol{G}_{\pi}(\boldsymbol{r})=\boldsymbol{0}$ or $\nabla \ln \phi^{e q \cdot}(\boldsymbol{r})=\nabla \ln p(\boldsymbol{r})$, predicts the equilibrium phase proportional to the probability density, $\phi^{e q} \cdot(\boldsymbol{r})=C p(\boldsymbol{r}), \varphi^{e q}=\varphi \exp \left(\mathrm{i}^{e q \cdot}\right)$, and hence $j\left[\varphi^{e q \cdot}\right]=C(\hbar / m) p \nabla p$. These two phase-transformed equilibrium states thus exhibit the same probability distribution but differ in their current densities.

One next introduces the current density of the resultant quantum entropy for the adopted choice of the phase-flux $\boldsymbol{J}_{\pi}(\boldsymbol{r})$ :

$$
\boldsymbol{J}_{s}(\boldsymbol{r})=F_{p}(\boldsymbol{r}) \boldsymbol{j}_{p}(\boldsymbol{r})+F_{\pi}(\boldsymbol{r}) \boldsymbol{J}_{\pi}(\boldsymbol{r}) .
$$

In the associated entropy-continuity equation,

$$
d s(\boldsymbol{r}) / d t \equiv \dot{s}(\boldsymbol{r})=\sigma_{s}(\boldsymbol{r})=\partial s(\boldsymbol{r}) / \partial t+\nabla \cdot \boldsymbol{J}_{s}(\boldsymbol{r}),
$$

its divergence $\nabla \cdot \boldsymbol{J}_{S}(\boldsymbol{r})$ determines the corresponding entropy inflow to the infinitesimal region around $\boldsymbol{r}$. The first term in the right-hand side of the preceding equation is suggested by the entropy differential, 


$$
\begin{aligned}
d S[p, \pi] & =\int \frac{\partial s(\boldsymbol{r})}{\partial p(\boldsymbol{r})} d p(\boldsymbol{r}) d \boldsymbol{r}+\int \frac{\partial s(\boldsymbol{r})}{\partial \pi(\boldsymbol{r})} d \pi(\boldsymbol{r}) d \boldsymbol{r} \\
& =\int\left[F_{p}(\boldsymbol{r}) d p(\boldsymbol{r})+F_{\pi}(\boldsymbol{r}) d \pi(\boldsymbol{r})\right] d \boldsymbol{r}, \\
\partial s(\boldsymbol{r}) / \partial t & =F_{p}(\boldsymbol{r}) \frac{\partial p(\boldsymbol{r})}{\partial t}+F_{\pi}(\boldsymbol{r}) \frac{\partial \pi(\boldsymbol{r})}{\partial t},
\end{aligned}
$$

while the divergence of the entropy current generally gives:

$$
\begin{aligned}
\nabla \cdot \boldsymbol{J}_{s} & =\left(\nabla F_{p}\right) \cdot \boldsymbol{j}_{p}+\left(\nabla F_{\pi}\right) \cdot \boldsymbol{J}_{\pi}+F_{p} \nabla \cdot \boldsymbol{j}_{p}+F_{\pi} \nabla \cdot \boldsymbol{J}_{\pi} \\
& =\boldsymbol{G}_{p} \cdot \boldsymbol{j}_{p}+\boldsymbol{G}_{\pi} \cdot \boldsymbol{J}_{\pi}+F_{p} \nabla \cdot \boldsymbol{j}_{p}+F_{\pi} \nabla \cdot \boldsymbol{J}_{\pi} .
\end{aligned}
$$

When combined with the probability- and phase-continuity equations these relations give the following, thermodynamic-like expression for the rate of the local production of the resultant quantum entropy:

$$
\sigma_{s}(\boldsymbol{r})=\boldsymbol{G}_{p}(\boldsymbol{r}) \cdot \boldsymbol{j}_{p}(\boldsymbol{r})+\boldsymbol{G}_{\pi}(\boldsymbol{r}) \cdot \boldsymbol{J}_{\pi}(\boldsymbol{r})+F_{\pi}(\boldsymbol{r}) \sigma_{\pi}(\boldsymbol{r}) .
$$

This expression simplifies for the absolute definitions of the phase-source and phase-current concepts [Eqs. (39a, 39a)]. In the former the second term of the preceding equation vanishes:

$$
\sigma_{s}(\boldsymbol{r})=\boldsymbol{G}_{p}(\boldsymbol{r}) \cdot \boldsymbol{j}_{p}(\boldsymbol{r})+F_{\pi}(\boldsymbol{r}) \sigma_{\pi}^{a b s .}(\boldsymbol{r}) .
$$

Therefore, this local production of the resultant quantum entropy vanishes in the strong-stationary state $\varphi_{j}, \sigma_{s}\left[\varphi_{j} ; \boldsymbol{r}\right]=0$, e.g., in the nondegenerate ground state of a molecule for which $\phi\left[\varphi_{j}\right]=0, \boldsymbol{j}_{p}(\boldsymbol{r})=\boldsymbol{0}$ and $\sigma_{\pi}\left[\varphi_{j}\right]=0$.

The second absolute reference gives the truly thermodynamic expression, solely in terms of products of the affinities and conjugate fluxes,

$$
\sigma_{s}(\boldsymbol{r})=\boldsymbol{G}_{p}(\boldsymbol{r}) \cdot \boldsymbol{j}_{p}(\boldsymbol{r})+\boldsymbol{G}_{\pi}(\boldsymbol{r}) \cdot \boldsymbol{J}_{\pi}^{a b s .}(\boldsymbol{r}) .
$$

This definition indeed implies that zero affinities give rise to vanishing source of the quantum entropy, as is the case in the ordinary irreversible thermodynamics [32].

\section{Promolecule to molecule transition}

This phenomenological IT treatment allows one to tackle interesting chemical problems involving specific equilibrium relaxations in the electronic structure of molecules and their constituent fragments. As an illustration let us examine a local estimate of the time required for the equilibrium promolecule $\rightarrow$ molecule relaxation in the oneelectron molecular system, e.g., the prototype covalent half-bond of $\mathrm{H}_{2}^{+} \equiv \mathrm{A}-\mathrm{B}$. One invisages an equilibrium transition between the equilibrium state $\phi_{e q}^{0} .(\boldsymbol{r})$, for the promolecular phase $\varphi_{\text {eq. }}^{0}(\boldsymbol{r})=-(1 / 2) \ln p^{0}(\boldsymbol{r})$ determined by the probability density $p^{0}=\left(p_{0}^{\mathrm{A}}+p_{0}^{\mathrm{B}}\right) / 2$ of the (equally weighted) ground-state distributions of the isolated hydrogen atoms, and the equilibrium molecular state $\varphi_{e q} .(\boldsymbol{r})$, exhibiting the phase 
$\phi_{\text {eq. }} .(\boldsymbol{r})=-(1 / 2) \ln p_{0}(\boldsymbol{r})$ specified by the molecular ground-state probability density $p_{0}$. Even a local estimate of this quantity would be of great value for both the structural chemistry and reactivity theory, by allowing one to distinguish a fast (chemically "hard") and slow (chemically "soft") relaxing processes and regions in the molecular system under consideration. A relation between the regional chemical reactivity and its average relaxation time is also intriguing. One would be also interested in differences between such temporal descriptors of the bonding and nonbonding regions in a molecule.

In what follows we shall refer to the local relaxation in the molecular electronic structure, from the initial, variationally non-optimum promolecular density $p^{0}(\boldsymbol{r})$ at time $t_{0}(\boldsymbol{r}) \equiv 0$ to the variationally optimum molecular density $p_{0}(\boldsymbol{r})$, after the relaxation time $\tau(\boldsymbol{r})=t(\boldsymbol{r})-t_{0}(\boldsymbol{r})=t(\boldsymbol{r})$. In this transition the initial difference of the probability density, measuring a displacement from the relaxed distribution $p_{0}, \Delta p(\boldsymbol{r})=p^{0}(\boldsymbol{r})-p_{0}(\boldsymbol{r}) \equiv-g(\boldsymbol{r} ; 0)$, acts as the driving force (negative time "gradient") for this structure rearrangement. It effects the return of the system to the molecular equilibrium $p_{0}(\boldsymbol{r})$ at time $\tau(\boldsymbol{r})$, via the distribution spontaneous response $\delta p(\boldsymbol{r})=-\Delta p(\boldsymbol{r})$, the negative of the displacement from the equilibrium distribution, which relaxes the initial force to zero: $g(\boldsymbol{r} ; \tau)=0$.

We adopt the quadratic approximation, in which this probability "gradient" is linearly dependent on time:

$$
g(\boldsymbol{r} ; \tau)=g(\boldsymbol{r} ; 0)+H(\boldsymbol{r} ; 0) \tau, \quad H(\boldsymbol{r} ; 0)=\left.\frac{\partial g(\boldsymbol{r}, t)}{\partial t}\right|_{t_{0}} .
$$

It also determines the quadratic expansion at time $t=0$ of a density $F(\boldsymbol{r}, t)$ of the physical quantity called the probability "action", $[F]=[$ probability $\times$ time $]$,

$$
\begin{aligned}
& F=\int F(\boldsymbol{r}, t) d \boldsymbol{r}=\int\left\{\int g(\boldsymbol{r} ; t) d t\right\} d \boldsymbol{r} \\
& F(\boldsymbol{r}, t)-\left.F\left(\boldsymbol{r}, t_{0}\right) \equiv \Delta F(\boldsymbol{r}, t) \cong \frac{\partial F(\boldsymbol{r}, t)}{\partial t}\right|_{t_{0}} \tau(\boldsymbol{r})+\left.\frac{1}{2} \frac{\partial^{2} F(\boldsymbol{r}, t)}{\partial t^{2}}\right|_{t_{0}} \tau(\boldsymbol{r})^{2} \\
& \quad=g(\boldsymbol{r} ; 0) \tau(\boldsymbol{r})+1 / 2 H(\boldsymbol{r} ; 0) \tau(\boldsymbol{r})^{2}
\end{aligned}
$$

determined by its (time-independent) "gradient" $g(\boldsymbol{r} ; 0) \equiv g(\boldsymbol{r})$ and "Hessian" $H(\boldsymbol{r} ; 0)$ $\equiv H(\boldsymbol{r})$ of Eq. (52). These local derivatives at $t_{0}(\boldsymbol{r})=0$ also determine the approximate time-dependent gradient of Eq. (52). The latter eventually vanishes when the molecular equilibrium is finally reached, after the "relaxation" time

$$
\tau(\boldsymbol{r})=-g(\boldsymbol{r}) / H(\boldsymbol{r})
$$

The local time-dependence of the system electron probability density $p(\boldsymbol{r}, t)$ is determined by the probability continuity [Eqs. (22) and (23)]. For the equilibrium electronic states at each stage of this transition [see Eq. (44)],

$$
\partial p(\boldsymbol{r}, t) / \partial t=-\nabla \cdot \boldsymbol{j}_{e q .}(\boldsymbol{r}, t)=\frac{\hbar}{m} \nabla \cdot\left[p(\boldsymbol{r}, t) \nabla \phi_{e q .}(\boldsymbol{r}, t)\right]=\frac{\hbar}{2 m} \nabla^{2} p(\boldsymbol{r}, t) .
$$


Therefore, the Laplacian of the density determines the rate of the equilibrium evolution of the local probability density in time. For the stationary equilibrium distribution $p(\boldsymbol{r} ; t)=p(\boldsymbol{r})$, and hence the time-independent Laplacian $\nabla^{2} p(\boldsymbol{r}, t)=\nabla^{2} p(\boldsymbol{r})$, the integration of the preceding equation over time expresses the relaxation time $\tau(\boldsymbol{r})$ in terms of the initial probability displacement $\Delta p(\boldsymbol{r})$ :

$$
\int_{t_{0}(\boldsymbol{r})}^{t_{0}(\boldsymbol{r})+\tau(\boldsymbol{r})} \frac{\partial p(\boldsymbol{r}, t)}{\partial t} d t=p(\boldsymbol{r} ; \tau)-p(\boldsymbol{r} ; 0)=-\Delta p(\boldsymbol{r}, \tau)=\delta p(\boldsymbol{r}, \tau)=\frac{\hbar \tau(\boldsymbol{r})}{2 m} \nabla^{2} p(\boldsymbol{r}) .
$$

The Laplacian of the promolecular probability distribution $p^{0}(\boldsymbol{r})$ can be thus used to estimate the promolecule $\rightarrow$ molecule local relaxation time $\tau(\boldsymbol{r})$. This (promolecular) integration over time required for the electron distribution to evolve, in the horizontalequilibrium fashion, from the (nonequilibrium) promolecular $p^{0}(\boldsymbol{r})\left(t_{0}=0\right)$ to (equilibrium) molecular $p_{0}(\boldsymbol{r})[t=\tau(\boldsymbol{r})]$ density, respectively, identifies the relevant promolecular time gradient and Hessian:

$$
\begin{aligned}
\int_{0}^{\tau(\boldsymbol{r})} \frac{\partial p(\boldsymbol{r}, t)}{\partial t} d t & =\frac{\hbar \tau(\boldsymbol{r})}{2 m} \nabla^{2} p^{0}(\boldsymbol{r})=p_{0}(\boldsymbol{r})-p^{0}(\boldsymbol{r}) \equiv-\Delta p(\boldsymbol{r}) \quad \text { or } \\
\tau(\boldsymbol{r}) & =-\frac{2 m \Delta p(\boldsymbol{r})}{\hbar \nabla^{2} p^{0}(\boldsymbol{r})} \equiv \frac{-g^{0}(\boldsymbol{r})}{H^{0}(\boldsymbol{r})}, \quad g^{0}(\boldsymbol{r})=\Delta p(\boldsymbol{r}), \\
H^{0}(\boldsymbol{r}) & =\partial^{2} F^{0}(\boldsymbol{r} ; t) /\left.\partial t^{2}\right|_{t=0}=\partial g^{0}(\boldsymbol{r} ; t) /\left.\partial t\right|_{t=0}=\frac{\hbar \nabla^{2} p^{0}(\boldsymbol{r})}{2 m} .
\end{aligned}
$$

Here, $g^{0}(\boldsymbol{r})$ and $H^{0}(\boldsymbol{r})$ stand for the local promolecular time gradient and Hessian, respectively, which determine the second-order change in the probability action [Eqs. (53), (54)]:

$$
\Delta^{(1+2)} F^{0}(\boldsymbol{r} ; t)=g^{0}(\boldsymbol{r}) \tau(\boldsymbol{r})+(1 / 2) H^{0}(\boldsymbol{r})[\tau(\boldsymbol{r})]^{2} .
$$

The relaxation time $\tau(\boldsymbol{r})$ then gives rise to the vanishing local probability force after this interval:

$$
g^{0}[\tau(\boldsymbol{r})]=g^{0}(\boldsymbol{r})+H^{0}(\boldsymbol{r}) \tau(\boldsymbol{r})=0 .
$$

Alternatively, by taking the molecular density $p_{0}(\boldsymbol{r})$ as the expansion starting point, for the initial time $t=\tau(\boldsymbol{r})$, the promolecular (displaced) distribution $p^{0}(\boldsymbol{r})$ is reached via the reverse molecule $\rightarrow$ promolecule propagation to time $t=0$. This (molecular) time integration gives:

$$
\begin{aligned}
\int_{\tau(\boldsymbol{r})}^{0} \frac{\partial p(\boldsymbol{r}, t)}{\partial t} d t & =\frac{\hbar[-\tau(\boldsymbol{r})]}{2 m} \nabla^{2} p_{0}(\boldsymbol{r})=p^{0}(\boldsymbol{r})-p_{0}(\boldsymbol{r}) \equiv \Delta p(\boldsymbol{r}) \quad \text { or } \\
\tau(\boldsymbol{r}) & =\frac{-2 m \Delta p(\boldsymbol{r})}{\hbar \nabla^{2} p_{0}(\boldsymbol{r})} \equiv \frac{-g_{0}(\boldsymbol{r})}{H_{0}(\boldsymbol{r})}, \quad g_{0}(\boldsymbol{r})=\Delta p(\boldsymbol{r})=g^{0}(\boldsymbol{r}),
\end{aligned}
$$




$$
H_{0}(\boldsymbol{r})=\partial^{2} F_{0}(\boldsymbol{r} ; t) /\left.\partial t^{2}\right|_{t=\tau}=\partial g_{0}(\boldsymbol{r} ; t) /\left.\partial t\right|_{t=\tau}=\frac{\hbar \nabla^{2} p_{0}(\boldsymbol{r})}{2 m} .
$$

This transition generates the molecular quadratic expansion of the probability-action density,

$$
\Delta^{(1+2)} F_{0}(\boldsymbol{r} ; t)=-g_{0}[\tau(\boldsymbol{r})] \tau(\boldsymbol{r})+(1 / 2) H_{0}(\boldsymbol{r})[\tau(\boldsymbol{r})]^{2}=(1 / 2) H_{0}(\boldsymbol{r})[\tau(\boldsymbol{r})]^{2},
$$

where we have recognized the vanishing molecular time-gradient: $g_{0}[\tau(\boldsymbol{r})]=0$.

Therefore, Eqs. (58) and (61) give rise to the consistency condition,

$$
\tau(\boldsymbol{r})=\frac{-g^{0}(\boldsymbol{r})}{H_{0}(\boldsymbol{r})}=\frac{-g^{0}(\boldsymbol{r})}{H^{0}(\boldsymbol{r})},
$$

yielding the unbiased estimate of the local relaxation time,

$$
\tau(\boldsymbol{r})=-\left[g^{0}(\boldsymbol{r}) / 2\right]\left[H_{0}(\boldsymbol{r})^{-1}+H^{0}(\boldsymbol{r})^{-1}\right] \equiv-g^{0}(\boldsymbol{r}) / H(\boldsymbol{r}),
$$

for the transition-state probability Hessian $H(\boldsymbol{r})=H_{h}(\boldsymbol{r})$ representing the harmonic $(h)$ average (reduced) value resulting from the promolecular and molecular estimates.

To summarize, the local transition time,

$$
\begin{gathered}
\tau(\boldsymbol{r})=\frac{-m \Delta p(\boldsymbol{r})}{\hbar}\left(\frac{1}{\nabla^{2} p^{0}(\boldsymbol{r})}+\frac{1}{\nabla^{2} p_{0}(\boldsymbol{r})}\right) \equiv-\frac{2 m \Delta p(\boldsymbol{r})}{\left.\hbar \nabla^{2} p(\boldsymbol{r})\right|_{h}}=-g^{0}(\boldsymbol{r}) / H_{h}(\boldsymbol{r}), \\
H_{h}(\boldsymbol{r})=\frac{2 H^{0}(\boldsymbol{r}) H_{0}(\boldsymbol{r})}{H^{0}(\boldsymbol{r})+H_{0}(\boldsymbol{r})}=\frac{\left.\hbar \nabla^{2} p(\boldsymbol{r})\right|_{h}}{2 m},\left.\quad \nabla^{2} p(\boldsymbol{r})\right|_{h}=\frac{2\left[\nabla^{2} p^{0}(\boldsymbol{r})\right]\left[\nabla^{2} p_{0}(\boldsymbol{r})\right]}{\nabla^{2} p^{0}(\boldsymbol{r})+\nabla^{2} p_{0}(\boldsymbol{r})},
\end{gathered}
$$

is thus determined by the harmonic average of the density Laplacian in the harmonic time Hessian $H_{h}(\boldsymbol{r})$, corresponding to the transition-state between these two extreme electron configurations. To avoid a negative time predictions in this harmonic approximation, one could adopt the modulus of this local time estimate. These predictions can be subsequently averaged over the whole physical space or its selected domains. A natural space-average (global) value of this local relaxation time is obtained by using the molecular probabilities as weights in the associated mean-value expression:

$$
\tau=\int p_{0}(\boldsymbol{r}) \tau(\boldsymbol{r}) d \boldsymbol{r} .
$$

As an illustration consider the prototype covalent half-bond in $\mathrm{H}_{2}^{+}$, in the minimum basis set description of two $1 s$ AO contributed by the constituent hydrogens, for the fixed internuclear distance $R$ (atomic units are used throughout):

$\chi \mathrm{X}\left(r_{\mathrm{X}}\right)=1 s_{\mathrm{X}}\left(r_{\mathrm{X}}\right)=\pi^{-1 / 2} \exp \left(-r_{\mathrm{X}}\right), \quad r_{\mathrm{X}}=\left|\boldsymbol{r}_{\mathrm{X}}(R)\right| \equiv\left|\boldsymbol{r}-\boldsymbol{R}_{\mathrm{X}}(R)\right|, \quad \mathrm{X}=\mathrm{A}, \mathrm{B}$. 
In the ground-state the system electron occupies the bonding MO

$$
\varphi_{b}(\boldsymbol{r})=\{2[1+S(R)]\}^{-1 / 2}\left[\chi_{\mathrm{A}}\left(r_{\mathrm{A}}\right)+\chi_{\mathrm{B}}\left(r_{\mathrm{B}}\right)\right] \equiv \mathcal{N}(R)\left[\chi_{\mathrm{A}}\left(r_{\mathrm{A}}\right)+\chi_{\mathrm{B}}\left(r_{\mathrm{B}}\right)\right],
$$

where the normalization constant $\mathcal{N}(R)$ depends on the overlap integral

$$
S(R)=\left\langle\chi_{\mathrm{A}} \mid \chi_{\mathrm{B}}\right\rangle=\mathrm{e}^{-R}\left(1+R+R^{2} / 3\right)
$$

The two (spherical) densities of constituent atoms,

$$
p_{\mathrm{X}}\left(r_{\mathrm{X}}\right)=\left[\chi_{\mathrm{X}}\left(r_{\mathrm{X}}\right)\right]^{2}=\pi^{-1} \exp \left(-2 r_{\mathrm{X}}\right), \quad \mathrm{X}=\mathrm{A}, \mathrm{B},
$$

determine the symmetrical (promolecular) reference distribution $p^{0}(\boldsymbol{r})=\left[p_{\mathrm{A}}\left(\boldsymbol{r}_{\mathrm{A}}\right)+\right.$ $\left.p_{\mathrm{B}}\left(\boldsymbol{r}_{\mathrm{B}}\right)\right] / 2$. They also generate the atomic Laplacians $\left\{\nabla^{2} p_{\mathrm{X}}\left(r_{\mathrm{X}}\right)=(4 / \pi)(1-\right.$ $\left.\left.1 / r_{\mathrm{X}}\right) \exp \left(-2 r_{\mathrm{X}}\right)\right\}$ and hence the Laplacian of the promolecular probability distribution:

$$
\nabla^{2} p^{0}(\boldsymbol{r})=\left[\nabla^{2} p_{\mathrm{A}}\left(r_{\mathrm{A}}\right)+\nabla^{2} p_{\mathrm{B}}\left(r_{\mathrm{B}}\right)\right] / 2 .
$$

The molecular probability density is similarly generated by the occupied MO:

$$
p_{0}(\boldsymbol{r})=\left[\varphi_{b}(\boldsymbol{r})\right]^{2}=\mathcal{N}^{2}(R)\left[p_{\mathrm{A}}\left(r_{\mathrm{A}}\right)+p_{\mathrm{B}}\left(r_{\mathrm{B}}\right)+2 \chi_{\mathrm{A}}\left(r_{\mathrm{A}}\right) \chi_{\mathrm{B}}\left(r_{\mathrm{B}}\right)\right] \equiv p_{0}\left(r_{\mathrm{A}}, r_{\mathrm{B}}\right)
$$

It generates the associated molecular Laplacian

$$
\begin{aligned}
& \nabla^{2} p_{0}\left(r_{\mathrm{A}}, r_{\mathrm{B}}\right)=\mathcal{N}^{2}(R)\left\{\nabla^{2} p_{\mathrm{A}}\left(r_{\mathrm{A}}\right)+\nabla^{2} p_{\mathrm{B}}\left(r_{\mathrm{B}}\right)\right. \\
& \left.+\left[p_{\mathrm{A}}\left(r_{\mathrm{A}}\right) p_{\mathrm{B}}\left(r_{\mathrm{B}}\right)\right]^{1 / 2}\left[2\left(1+\boldsymbol{e}_{\mathrm{A}} \cdot \boldsymbol{e}_{\mathrm{B}}\right)-\sum_{\mathrm{X}=\mathrm{A}, \mathrm{B}} \nabla \cdot \boldsymbol{e}_{\mathrm{X}}\right]\right\},
\end{aligned}
$$

where the unit vector $\boldsymbol{e}_{\mathrm{X}}=\boldsymbol{r}_{\mathrm{X}} / r_{\mathrm{X}}$.

Consider now three illustrative locations along the bond axis, for the collinear $\boldsymbol{r}_{\mathrm{A}}$ and $\boldsymbol{r}_{\mathrm{B}}$, when $\boldsymbol{e}_{\mathrm{A}}=-\boldsymbol{e}_{\mathrm{B}}$ : in the bonding region, at the bond midpoint, $\boldsymbol{r}=\boldsymbol{r}_{m}$, when $r_{\mathrm{A}}=r_{\mathrm{B}}=R / 2$, in the position of one nucleus, say $\boldsymbol{r} \rightarrow \boldsymbol{R}_{\mathrm{A}}$, and in the nonbonding part of orbital $\chi_{\mathrm{A}}, \boldsymbol{r}=\boldsymbol{r}_{n}$ for $r_{\mathrm{A}}=R / 2$ and $r_{\mathrm{B}}=3 R / 2$. These axial positions remove the contribution to the molecular Hessian due to the overlap between two atomic densities. For $R=2 \approx R_{e}$, these locations give the following probability differences (time-gradients):

$$
\begin{array}{ll}
p_{0}\left(\boldsymbol{r}_{m}\right)=1.261 p_{\mathrm{A}}\left(\boldsymbol{r}_{m}\right)>p^{0}\left(\boldsymbol{r}_{m}\right)=p_{\mathrm{A}}\left(\boldsymbol{r}_{m}\right)=0.0431 & \text { or } g\left(\boldsymbol{r}_{m}\right)=-0.011 \\
p_{0}\left(\boldsymbol{R}_{\mathrm{A}}\right)=0.129<p^{0}\left(\boldsymbol{R}_{\mathrm{A}}\right)=0.162 & \text { or } g\left(\boldsymbol{R}_{\mathrm{A}}\right)=0.033 \\
p_{0}\left(\boldsymbol{r}_{n}\right)=0.035>p^{0}\left(\boldsymbol{r}_{n}\right)=0.029 & \text { or } g\left(\boldsymbol{r}_{n}\right)=-0.006
\end{array}
$$


The bonding electron position gives the molecular Laplacian

$$
\begin{aligned}
\nabla^{2} p_{0}\left(\boldsymbol{r}_{m}\right) & =\{2 /[1+S(R)]\}\left[p_{\mathrm{A}}\left(\boldsymbol{r}_{m}\right)+p_{\mathrm{B}}\left(\boldsymbol{r}_{m}\right)\right](1-2 / R) \\
& =\{4 /[1+S(R)]\} p_{\mathrm{A}}(R / 2)(1-2 / R) .
\end{aligned}
$$

It vanishes for $R=2, \nabla^{2} p_{0}\left[\boldsymbol{r}_{m}(R=2)\right]=\nabla^{2} p^{0}\left[\boldsymbol{r}_{m}(R=2)\right]=H((R=2)=0$, thus predicting an infinite value of $\tau\left[\boldsymbol{r}_{m}(R=2)\right] \rightarrow \infty$ close to the equilibrium bond length of $\mathrm{H}_{2}^{+}, R_{e}=1.997$, for which the dissociation energy $D_{e}=0.103$. It reflects an infinitely "soft" character in the middle of the bonding region. In the nuclear-cusp position the Laplacian diverges due to $\nabla^{2} p_{\mathrm{A}}\left(r_{\mathrm{A}} \rightarrow 0\right) \rightarrow-\infty$, thus predicting $\tau\left(\boldsymbol{R}_{\mathrm{A}}\right) \rightarrow 0$, i.e., an infinitely "hard" electron location in $\mathrm{H}_{2}^{+}$. Finally, for the nonbonding location one obtains $H\left(\boldsymbol{r}_{n}\right)=0.001$ and hence a finite estimate of the local relaxation time $\tau\left(\boldsymbol{r}_{n}\right) \approx 7$.

\section{Ground and excited configurations of $\pi$-electrons in benzene}

The phase/current aspect of the molecular electronic structure gains an extra significance in the domain of degenerate electronic states. As an illustrative example let us consider excited configurations of the $\pi$-electron system in benzene, within the familiar Hückel approximation of the LCAO MO theory, consisting of $N_{\pi}=6$ valence $2 p_{z}$-electrons contributed by the ring carbons. All $\pi$-MO's are then expanded in the minimum basis set comprising of the $\chi \equiv 2 p_{z}$ AO contributed by the six ring carbons,

$$
\begin{aligned}
\left\{\chi_{k}(\boldsymbol{r})\right. & \left.=\mathcal{N} z_{k} \exp \left(-\alpha r_{k}\right), \quad r_{k}=\left|\boldsymbol{r}-\boldsymbol{R}_{k}\right|, \quad k=1,2, \ldots, 6\right\}, \\
\pi_{s}(\boldsymbol{r}) & =\sum_{k} C_{k, s} \chi_{k}\left(\boldsymbol{r}_{k}\right), \quad s=1,2, \ldots, 6
\end{aligned}
$$

where $\mathcal{N}=\langle\chi \mid \chi\rangle^{-1 / 2}$ is the AO-normalization constant and $\boldsymbol{R}_{k}=\left(\boldsymbol{i} X_{k}+\boldsymbol{j} Y_{k}+\boldsymbol{k} Z_{k}\right)$ stands for the fixed position of $k$ th carbon nucleus, with the AO axes perpendicular to the molecular $\left(x, y ; Z_{k}=0\right)$ plane: $z_{k}=z-Z_{k}=z$.

One recalls that all $\pi$-MO in benzene are completely determined by the ring symmetry, with the three lowest (doubly-occupied) AO combinations including the (nondegenerate) normalized sum of all basis functions,

$$
\pi_{1}(\boldsymbol{r})=(1 / 6)^{1 / 2} \sum_{k} \chi_{k}\left(\boldsymbol{r}_{k}\right), \quad \varepsilon_{1}=\alpha+2 \beta,
$$

and two degenerate (real) MO's:

$$
\begin{aligned}
& \pi_{2}(\boldsymbol{r})=(1 / 3)^{1 / 2} \sum_{k} \cos (\pi k / 3) \chi_{k}\left(\boldsymbol{r}_{k}\right), \\
& \pi_{3}(\boldsymbol{r})=(1 / 3)^{1 / 2} \sum_{k} \sin (\pi k / 3) \chi_{k}\left(\boldsymbol{r}_{k}\right), \quad \varepsilon_{2}=\varepsilon_{3}=\alpha+\beta
\end{aligned}
$$


where the MO energies $\left\{\varepsilon_{s}\right\}$ are expressed in terms of the familiar Coulomb $(\alpha<0)$ and resonance $(\beta<0)$ integrals of carbon atoms in the Hückel theory.

The two MO of Eq. (77) can be interpreted as the real (Re) and imaginary (Im) parts of their equivalent linear combinations $\varphi_{1}(\boldsymbol{r})$ and $\varphi_{1}(\boldsymbol{r})^{*}$ exhibiting complex expansion coefficients:

$$
\begin{aligned}
& \pi_{2}(\boldsymbol{r})=(1 / 2)\left[\varphi_{1}(\boldsymbol{r})+\varphi_{1}^{*}(\boldsymbol{r})\right]=\operatorname{Re}[\varphi(\boldsymbol{r})] \text { and } \\
& \pi_{3}(\boldsymbol{r})=[1 /(2 \mathrm{i})]\left[\varphi_{1}(\boldsymbol{r})-\varphi_{1}^{*}(\boldsymbol{r})\right]=\operatorname{Im}[\varphi(\boldsymbol{r})],
\end{aligned}
$$

where

$$
\begin{aligned}
\varphi_{1}(\boldsymbol{r}) & \equiv R_{1}(\boldsymbol{r}) \exp \left[\mathrm{i} \Phi_{1}(\boldsymbol{r})\right]=(1 / 2)^{1 / 2}\left[\pi_{2}(\boldsymbol{r})+\mathrm{i} \pi_{3}(\boldsymbol{r})\right] \\
& =(1 / 6)^{1 / 2} \sum_{k}[\cos (\pi k / 3)+\mathrm{i} \sin (\pi k / 3)] \chi_{k}\left(\boldsymbol{r}_{k}\right) \\
& =(1 / 6)^{1 / 2} \sum_{k} \exp [\mathrm{i}(\pi k / 3)] \chi_{k}\left(\boldsymbol{r}_{k}\right) \\
& \equiv(1 / 6)^{1 / 2} \sum_{k} \exp \left(\mathrm{i} \phi_{k}\right) \chi_{k}\left(\boldsymbol{r}_{k}\right) \equiv \sum_{k} \zeta_{k} \chi_{k}\left(\boldsymbol{r}_{k}\right)
\end{aligned}
$$

where $R_{1}(\boldsymbol{r})$ and $\Phi_{1}(\boldsymbol{r})$ stand for the resultant modulus and phase functions of $\varphi_{1}(\boldsymbol{r})$. These complex MO are also eigenfunctions of the $\pi$-electron Hamiltonian with the same eigenvalue $\varepsilon_{2}=\varepsilon_{3}$. Thus, the state vector $\varphi_{1}(\boldsymbol{r})$ of Eq. (79) and its complex conjugate

$$
\begin{aligned}
\varphi_{1}^{*}(\boldsymbol{r}) & \equiv R_{1}(\boldsymbol{r}) \exp \left[-\mathrm{i} \Phi_{1}(\boldsymbol{r})\right]=(1 / 2)^{1 / 2}\left[\pi_{2}(\boldsymbol{r})-\mathrm{i} \pi_{3}(\boldsymbol{r})\right] \\
& =(1 / 6)^{1 / 2} \sum_{k}[\cos (\pi k / 3)-\mathrm{i} \sin (\pi k / 3)] \chi_{k}\left(\boldsymbol{r}_{k}\right) \\
& =(1 / 6)^{1 / 2} \sum_{k} \exp [-\mathrm{i}(\pi k / 3)] \chi_{k}\left(\boldsymbol{r}_{k}\right) \\
& =(1 / 6)^{1 / 2} \sum_{k} \exp \left(-\mathrm{i} \phi_{k}\right) \chi_{k}\left(\boldsymbol{r}_{k}\right) \equiv \sum_{k} \zeta_{k}^{*} \chi_{k}\left(\boldsymbol{r}_{k}\right)
\end{aligned}
$$

are the (local) unitary transforms of the original (orthonormal) MO,

$$
\left[\varphi_{1}, \varphi_{1}^{*}\right]=\left[\pi_{2}, \pi_{3}\right] \frac{1}{\sqrt{2}}\left[\begin{array}{cc}
1 & 1 \\
i & -i
\end{array}\right]=\left[\pi_{2}, \pi_{3}\right] \mathbf{U}, \quad \mathbf{U U}^{\dagger}=\mathbf{I},
$$

and, together with the lowest orbital $\pi_{1}$, they give rise to the same one-determinantal electronic state of $\pi$-electrons as do the original (real) doubly-occupied MO:

$$
\begin{aligned}
\Psi_{\pi} & =\operatorname{det}\left[\left(\pi_{1}^{+}, \pi_{1}^{-}\right),\left(\pi_{2}^{+}, \pi_{2}^{-}\right),\left(\pi_{3}^{+}, \pi_{3}^{-}\right)\right] \\
& =\operatorname{det}\left[\left(\pi_{1}^{+}, \pi_{1}^{-}\right),\left(\varphi_{1}^{+}, \varphi_{1}^{-}\right),\left(\varphi_{1}^{*+}, \varphi_{1}^{*-}\right)\right] \equiv \Psi_{0} .
\end{aligned}
$$


In this notation the upper indices $(+,-)$ of MO defining the Slater determinant denote the alternative spin states of an electron: $\{|\sigma\rangle\}=\{|\hbar / 2\rangle,|-\hbar / 2\rangle\} \equiv\{|+\rangle,|-\rangle\}$.

To summarize, the degenerate complex $\pi$-MO [Eqs. (79), (80)] are linear combinations of the real basis functions with complex LCAO MO coefficients $\left\{\zeta_{k}=\right.$ $\left.R_{k} \exp \left(i \phi_{k}\right)\right\}$ and $\left\{\zeta_{k}^{*}=R_{k} \exp \left(-i \phi_{k}\right)\right\}$, respectively, characterized by the AO phases $\left\{\phi_{k}=\pi k / 3\right\}$ and equal moduli $\left\{R_{k}=(1 / 6)^{1 / 2}\right\}$. The expansion coefficients $\mathbf{Z}=\left\{Z_{k, s}\right\}$ of the ground-state occupied equivalent MO

$$
\varphi_{s} \in\left(\pi_{1}, \varphi_{1}, \varphi_{1}^{*}\right) \equiv \sum_{k} Z_{k, s} \chi_{k},
$$

where $Z_{k, 1}=C_{k, 1}=(1 / 6)^{1 / 2}, Z_{k, 2}=\zeta_{k}$ and $Z_{k, 3}=\zeta_{k}^{*}$, define the (symmetric, real) 1 -electron density matrix in $\mathrm{AO}$ representation, also called the Charge and Bond-Order (CBO) matrix

$$
\begin{aligned}
\gamma\left(\Psi_{0}\right) & =2 \mathbf{Z}\left(\Psi_{0}\right) \mathbf{Z}\left(\Psi_{0}\right)^{\dagger}=\left\{\gamma_{k, l}\left(\Psi_{0}\right)=\sum_{s} Z_{k, s}\left(\Psi_{0}\right)^{*} n_{s}\left(\Psi_{0}\right) Z_{l, s}\left(\Psi_{0}\right)\right. \\
& =(1+\exp \{\mathrm{i}[\pi(l-k) / 3]\}+\exp \{\mathrm{i}[\pi(k-l) / 3]\}) / 3=(1+2 \cos [\pi(l-k) / 3]) / 3\} .
\end{aligned}
$$

Its elements are summarized by the representative diagonal and off-diagonal predictions:

$$
\gamma_{k, k}\left(\Psi_{0}\right)=1, \quad \gamma_{k, k+1}\left(\Psi_{0}\right)=2 / 3, \quad \gamma_{k, k+2}\left(\Psi_{0}\right)=0, \quad \gamma_{k, k+3}\left(\Psi_{0}\right)=-1 / 3
$$

Their signs indicate that $\Psi_{0}$, generating equal distribution of $\pi$-electrons $\left(\gamma_{k, k}\right)$, represents a strongly ortho-bonding $\left(\gamma_{k, k+1}\right)$, meta-nonbonding $\left(\gamma_{k, k+2}\right)$, and weakly para-antibonding $\left(\gamma_{k, k+3}\right)$ state of the ring $\pi$-electrons.

For the the expectation value of the local current of Eq. (4a), defined by the Hermitian operator of Eq. (4b), one then obtains the sum of the MO currents,

$$
\begin{aligned}
\boldsymbol{j}_{S}(\boldsymbol{r}) & =\left\langle\varphi_{s}|\hat{\mathbf{j}}(\boldsymbol{r})| \varphi_{s}\right\rangle=(\hbar / m) p_{s}(\boldsymbol{r}) \nabla \Phi_{s}(\boldsymbol{r}), \quad p_{s}(\boldsymbol{r})=R_{S}(\boldsymbol{r})^{2}, \quad \text { (86) } \\
\boldsymbol{j}(\boldsymbol{r}) \equiv \sum_{s} n_{s} \boldsymbol{j}_{s}(\boldsymbol{r}) & =\sum_{k} \sum_{l}\left\langle\chi_{k}|\hat{\mathbf{j}}(\boldsymbol{r})| \chi_{l}\right\rangle\left(\sum_{s} Z_{l, s}^{*} n_{s} Z_{k, s}\right) \\
& =\sum_{k} \sum_{l}\left\langle\chi_{k}|\hat{\mathbf{j}}(\boldsymbol{r})| \chi_{l}\right\rangle \gamma_{l, k} \equiv \sum_{k} \sum_{l} \boldsymbol{j}_{k, l}(\boldsymbol{r}) \gamma_{l, k} \\
& =\operatorname{tr}[\mathbf{j}(\boldsymbol{r}) \boldsymbol{\gamma}]=\operatorname{tr}\left[\gamma_{\mathbf{j}}(\boldsymbol{r})\right]=\sum_{k} \sum_{l} \frac{\hbar}{2 m \mathrm{i}}\left(\gamma_{k, l}-\gamma_{l, k}\right) \chi_{k}(\boldsymbol{r}) \nabla \chi_{l}(\boldsymbol{r})
\end{aligned}
$$




$$
\begin{aligned}
& =\frac{\hbar}{m} \sum \sum_{k \neq l}\left[\frac{1}{2 \mathrm{i}}\left(\gamma_{k, l}-\gamma_{k, l}^{*}\right)\right] \chi_{k}(\boldsymbol{r}) \nabla \chi_{l}(\boldsymbol{r}) \\
& =\frac{\hbar}{m} \sum \sum_{k \neq l} \operatorname{Im}\left(\gamma_{k, l}\right) \chi_{k}(\boldsymbol{r}) \nabla \chi_{l}(\boldsymbol{r}),
\end{aligned}
$$

where the (antisymmetric) AO matrix of the current operator

$\mathbf{j}(\boldsymbol{r})=\left\{\boldsymbol{j}_{k, l}(\boldsymbol{r})=\left\langle\chi_{k}|\hat{\mathbf{j}}(\boldsymbol{r})| \chi_{l}\right\rangle=\frac{\hbar}{2 m \mathrm{i}}\left[\chi_{k}(\boldsymbol{r}) \nabla \chi_{l}(\boldsymbol{r})-\chi_{l}(\boldsymbol{r}) \nabla \chi_{k}(\boldsymbol{r})\right]=-\boldsymbol{j}_{l, k}(\boldsymbol{r})\right\}$.

Here, we have used the Hermitian character of $\gamma$ and $n_{s}$ stands for the MO occupation number. This expression shows that strongly overlapping basis functions of the ortho-bonds, for which local contributions $\chi_{k}(\boldsymbol{r}) \nabla \chi_{k+1}(\boldsymbol{r})$ assume an appreciable magnitude, dominate the resultant electronic current. One further observes that for the real basis set the diagonal elements of the AO current matrix $\mathbf{j}(\boldsymbol{r})$ identically vanish, $\boldsymbol{j}_{k, k}(\boldsymbol{r})=\mathbf{0}$.

For the real (symmetric) CBO matrix, when $\gamma_{l, k}=\gamma_{k, l}$, the antisymmetry of Eq. (88) gives rise to an exact cancellation of the off-diagonal terms in the trace od Eq. (87):

$$
\gamma_{l, k} \boldsymbol{j}_{k, l}(\boldsymbol{r})+\gamma_{k, l} \boldsymbol{j}_{l, k}(\boldsymbol{r})=\gamma_{k, l}\left[\boldsymbol{j}_{k, l}(\boldsymbol{r})+\boldsymbol{j}_{l, k}(\boldsymbol{r})\right]=\mathbf{0}
$$

This does not take place in configurations generating the complex $\mathrm{CBO}$ matrix. Indeed, by putting $\gamma_{k, l} \equiv M_{k, l} \exp \left(i \phi_{k, l}\right), \phi_{k, l} \neq 0$, one obtains from the CBO Hermitian character $\left(\gamma_{k, l}\right)^{*}=M_{k, l} \exp \left(-i \phi_{k, l}\right)=\gamma_{l, k} \neq \gamma_{k, l}$. Thus, the cancellation of Eq. (89) then no longer holds, giving rise to a finite current of $\pi$-electrons in such open-shell configurations.

It should be recalled that the $\mathrm{CBO}$ matrix embodies the AO-representation of the (Hermitian) MO density-operator,

$$
\hat{\mathrm{P}}_{\mathrm{MO}}=\sum_{s}\left|\varphi_{s}\right\rangle p_{s}\left\langle\varphi_{s}\right|, \quad\left(\hat{\mathrm{P}}_{\mathrm{MO}}\right)^{\dagger}=\hat{\mathrm{P}}_{\mathrm{MO}}, \quad \gamma_{k, l}=N_{\pi}\left\langle\chi_{k}\left|\hat{\mathrm{P}}_{\mathrm{MO}}\right| \chi_{l}\right\rangle
$$

projecting out the subspace of the MO occupied in the electronic configuration in question. The latter is identified either by the MO occupation vector $\boldsymbol{n}=\left\{n_{s}\right\}$ or the associated MO-probabilities $\boldsymbol{p}=\left\{p_{s}=n_{s} / N_{\pi}\right\}$. Therefore, the CBO matrix itself is Hermitian, $\gamma_{l, k}=\left(\gamma_{k, l}\right)^{*}$, with real diagonal elements, $\gamma_{k, k}=\left(\gamma_{k, k}\right)^{*}$, but for the complex off-diagonal elements $\gamma_{l, k} \neq \gamma_{k, l}$.

Therefore, the average local current of $\pi$-electrons of the benzene ring in their non-degenerate ground-state identically vanishes. Indeed, all MO currents $\boldsymbol{j}_{S}(\boldsymbol{r})$ then automatically vanish, since the MO phase is purely time dependent, $\phi_{s}(\boldsymbol{r}, t)=\phi_{0}(t)=$ $-\omega_{0} t, \omega_{0}=E_{0} / \hbar$, and hence $\nabla \phi_{s}(\boldsymbol{r}, t)=0$. The same is true in the open-shell (excited) $\pi$-electron configurations, which populate equaly both components of the degenerate MO energy levels. For example, in the doubly excited (singlet) configuration 


$$
\Psi_{1}=\operatorname{det}\left[\left(\pi_{1}^{+}, \pi_{1}^{-}\right),\left(\varphi_{1}^{+}, \varphi_{1}^{*+}\right),\left(\varphi_{2}^{-}, \varphi_{2}^{*-}\right)\right]
$$

involving the doubly occupied $\pi_{1}$ and the singly occupied $\varphi_{1}$, its complex conjugate $\varphi_{1}^{*}$, and the corresponding pair $\left(\varphi_{2}, \varphi_{2}^{*}\right)$ of the degenerate (virtual) MO level,

$$
\begin{aligned}
\varphi_{2}(\boldsymbol{r}) & =(1 / 2)^{1 / 2}\left[\pi_{4}(\boldsymbol{r})+\mathrm{i} \pi_{5}(\boldsymbol{r})\right]=(1 / 6)^{1 / 2} \sum_{k}[\cos (2 \pi k / 3)+\mathrm{i} \sin (2 \pi k / 3)] \chi_{k}\left(\boldsymbol{r}_{k}\right) \\
& =(1 / 6)^{1 / 2} \sum_{k} \exp [\mathrm{i}(2 \pi k / 3)] \chi_{k}\left(\boldsymbol{r}_{k}\right) \equiv(1 / 6)^{1 / 2} \sum_{k} \exp \left(\mathrm{i} \phi_{k, 2}\right) \chi_{k}\left(\boldsymbol{r}_{k}\right) \\
\varepsilon_{4} & =\varepsilon_{5}=\alpha-\beta
\end{aligned}
$$

gives rise to the following element of the CBO matrix $\gamma\left(\Psi_{1}\right)$,

$$
\gamma_{k, l}\left(\Psi_{1}\right)=\{1+2 \cos [\pi(l-k) / 2] \cos [\pi(l-k) / 6]\} / 3 .
$$

It predicts the representative values:

$$
\gamma_{k, k}\left(\Psi_{1}\right)=1, \quad \gamma_{k, k+1}\left(\Psi_{1}\right)=1 / 3, \quad \gamma_{k, k+2}\left(\Psi_{1}\right)=0, \quad \gamma_{k, k+3}\left(\Psi_{1}\right)=1 / 3
$$

Their signs again indicate the weakly ortho-bonding, meta-nonbonding, and weakly para-bonding nature of this electronic configuration.

The complete set of the ground-state real and complex $\pi-\mathrm{MO}$,

$$
\left\{\varphi_{s} \in\left[\pi_{1},\left(\varphi_{1}, \varphi_{1}^{*}\right),\left(\varphi_{2}, \varphi_{2}^{*}\right), \pi_{6}\right]\right\}
$$

where the highest $\pi$-MO involves alternating signs of the LCAO MO coefficients at neighboring atoms,

$$
\pi_{6}(\boldsymbol{r})=(1 / 6)^{1 / 2} \sum_{k}(-1)^{k+1} \chi_{k}\left(\boldsymbol{r}_{k}\right), \quad \varepsilon_{6}=\alpha-2 \beta,
$$

defines a convenient reference frame for discussing the complex elements of the CBO matrix in all excited configurations of $\pi$-electron in benzene, although the real and complex $\pi$-MO are no longer physically equivalent in such states. However, keeping the "frozen" complex MO, equivalent in the ground state configuration, allows one to systematically monitor changes due to the electron excitations in the bond multiplicities (or "orders"), with respect to the reference ground state.

Another example of the symmetric population pattern of degenerate components represents the antibonding configuration involving six electron excitations from $\Psi_{0}$,

$$
\Psi_{2}=\operatorname{det}\left[\left(\varphi_{2}^{+}, \varphi_{2}^{-}\right),\left(\varphi_{2}^{*+}, \varphi_{2}^{*-}\right),\left(\pi_{6}^{+}, \pi_{6}^{-}\right)\right] .
$$

It also gives rise to the real CBO matrix $\gamma\left(\Psi_{2}\right)$,

$$
\gamma_{k, l}\left(\Psi_{2}\right)=\left\{(-1)^{k+l} 2 \cos [2 \pi(l-k) / 3]\right\} / 3
$$


generating the following matrix elements representing AO populations and the ortho-, meta-, and para-interactions in this extreme antibonding state of the benzene $\pi$-bond ring:

$$
\gamma_{k, k}\left(\Psi_{2}\right)=1, \quad \gamma_{k, k+1}\left(\Psi_{2}\right)=-2 / 3, \quad \gamma_{k, k+2}\left(\Psi_{1}\right)=0, \quad \gamma_{k, k+3}\left(\Psi_{1}\right)=1 / 3
$$

They indicate that this configuration exhibits the strongly ortho-antibonding, metanonbonding and weakly para-bonding character, opposite to that of $\Psi_{0}$.

Two more symmetric-population configurations,

$$
\begin{aligned}
& \Psi_{3}=\operatorname{det}\left[\left(\pi_{1}^{+}, \pi_{1}^{-}\right),\left(\varphi_{2}^{+}, \varphi_{2}^{-}\right),\left(\varphi_{2}^{*+}, \varphi_{2}^{*-}\right)\right] \quad \text { (quadruply excited) and } \\
& \Psi_{4}=\operatorname{det}\left[\left(\varphi_{1}^{+}, \varphi_{1}^{-}\right),\left(\varphi_{1}^{*+}, \varphi_{1}^{*-}\right),\left(\pi_{6}^{+}, \pi_{6}^{-}\right)\right] \quad \text { (doubly excited), }
\end{aligned}
$$

generate the following expressions for their (real) $\mathrm{CBO}$ matrix elements:

$$
\begin{aligned}
& \gamma_{k, l}\left(\Psi_{3}\right)=\{1+2 \cos [2 \pi(l-k) / 3]\} / 3 \text { and } \\
& \gamma_{k, l}\left(\Psi_{4}\right)=\left\{(-1)^{k+l}+2 \cos [\pi(l-k) / 3]\right\} / 3 .
\end{aligned}
$$

They give rise to the following predictions,

$$
\begin{aligned}
& \gamma_{k, k}\left(\Psi_{3}\right)=1, \quad \gamma_{k, k+1}\left(\Psi_{3}\right)=\gamma_{k, k+2}\left(\Psi_{3}\right)=0, \quad \gamma_{k, k+3}\left(\Psi_{3}\right)=1 \quad \text { and } \\
& \gamma_{k, k}\left(\Psi_{4}\right)=1, \quad \gamma_{k, k+1}\left(\Psi_{4}\right)=\gamma_{k, k+2}\left(\Psi_{4}\right)=0, \quad \gamma_{k, k+3}\left(\Psi_{4}\right)=-1 / 3 \text {, }
\end{aligned}
$$

which indicate that both these states are ortho- and meta-nonbonding. Notice, however, that $\Psi_{3}$ realizes the full strength of the weak cross-ring para-bond, while $\Psi_{4}$ remains weakly para-antibonding.

Of interest also is the $\pi_{1} \rightarrow \pi_{6}$ excitation generating

$$
\Psi_{5}=\operatorname{det}\left[\left(\varphi_{1}^{+}, \varphi_{1}^{-}\right),\left(\varphi_{1}^{*+}, \varphi_{1}^{*-}\right), \pi_{1}^{+}, \pi_{6}^{-}\right]
$$

for which

$$
\gamma_{k, l}\left(\Psi_{5}\right)=(2 / 3) \cos [\pi(l-k) / 3]+(1 / 6)\left[(-1)^{k+l}+1\right],
$$

and hence:

$$
\gamma_{k, k}\left(\Psi_{5}\right)=1, \quad \gamma_{k, k+1}\left(\Psi_{5}\right)=1 / 3, \quad \gamma_{k, k+2}\left(\Psi_{5}\right)=0, \quad \gamma_{k, k+3}\left(\Psi_{5}\right)=-2 / 3
$$

This configuration thus appears weakly ortho-bonding, meta-nonbonding, and strongly para-antibonding.

In a systematic study of the bond-order changes one should also consider three more configurations representing a symmetric population pattern:

$$
\Psi_{6}=\operatorname{det}\left[\left(\varphi_{2}^{+}, \varphi_{2}^{-}\right),\left(\varphi_{2}^{*+}, \varphi_{2}^{*-}\right), \pi_{1}^{+}, \pi_{6}^{-}\right],
$$


involving 5 excitations, the triply excited state

$$
\Psi_{7}=\operatorname{det}\left[\pi_{1}^{+}, \varphi_{1}^{+}, \varphi_{2}^{-}, \varphi_{1}^{*+}, \varphi_{2}^{*-}, \pi_{6}^{-}\right]
$$

and the quadruply excited configuration

$$
\Psi_{8}=\operatorname{det}\left[\left(\pi_{6}^{+}, \pi_{6}^{-}\right), \varphi_{1}^{+}, \varphi_{1}^{*+}, \varphi_{2}^{-}, \varphi_{2}^{*-}\right]
$$

These $\pi$-electron determinants generate the corresponding CBO matrix elements:

$$
\begin{aligned}
& \gamma_{k, l}\left(\Psi_{6}\right)=(2 / 3) \cos [2 \pi(l-k) / 3]+(1 / 6)\left[(-1)^{k+l}+1\right], \\
& \gamma_{k, l}\left(\Psi_{7}\right)=(1 / 3)\{\cos [\pi(l-k) / 3]+\cos [2 \pi(l-k) / 3]\}+(1 / 6)\left[(-1)^{k+l}+1\right], \\
& \gamma_{k, l}\left(\Psi_{8}\right)=(1 / 3)\left\{(-1)^{k+l}+\cos [\pi(l-k) / 3]+\cos [2 \pi(l-k) / 3]\right\} .
\end{aligned}
$$

They predict the following intra- and inter-carbon bond orders in the benzene ring:

$$
\begin{aligned}
& \gamma_{k, k}\left(\Psi_{6}\right)=1, \quad \gamma_{k, k+1}\left(\Psi_{6}\right)=-1 / 3, \quad \gamma_{k, k+2}\left(\Psi_{6}\right)=0, \quad \gamma_{k, k+3}\left(\Psi_{6}\right)=2 / 3 \\
& \gamma_{k, k}\left(\Psi_{7}\right)=1, \quad \gamma_{k, k+1}\left(\Psi_{7}\right)=0, \quad \gamma_{k, k+2}\left(\Psi_{7}\right)=0, \quad \gamma_{k, k+3}\left(\Psi_{7}\right)=0 ; \\
& \gamma_{k, k}\left(\Psi_{8}\right)=1, \quad \gamma_{k, k+1}\left(\Psi_{8}\right)=\gamma_{k, k+3}\left(\Psi_{8}\right)=-1 / 3, \quad \gamma_{k, k+2}\left(\Psi_{8}\right)=0 .
\end{aligned}
$$

Therefore, configuration $\Psi_{7}$ is truly nonbonding in both the ring and cross-ring interactions, $\Psi_{6}$ is weakly ortho-antibonding, meta-nonbonding and strongly para-bonding, while $\Psi_{8}$ exhibits a weak ortho- and para-antibonding character while remaining meta-nonbonding.

After this brief exploration of configurations exhibiting the symmetric-population of degenerate levels, let us examine selected nonsymmetric distributions of electrons among such doubly degenerate states, which can lead to the complex CBO matrices. Let us first examine the energetically most accessible single excitations from $\varphi_{1},\left(\varphi_{1} \rightarrow \varphi_{2}\right)$ and $\left(\varphi_{1} \rightarrow \varphi_{2}^{*}\right)$

$$
\begin{aligned}
\Psi_{9} & =\operatorname{det}\left[\left(\pi_{1}^{+}, \pi_{1}^{-}\right),\left(\varphi_{1}^{*+}, \varphi_{1}^{*-}\right), \varphi_{1}^{+}, \varphi_{2}^{-}\right] \text {and } \\
\Psi_{10} & =\operatorname{det}\left[\left(\pi_{1}^{+}, \pi_{1}^{-}\right),\left(\varphi_{1}^{*+}, \varphi_{1}^{*-}\right), \varphi_{1}^{+}, \varphi_{2}^{*-}\right] .
\end{aligned}
$$

The representative $\mathrm{CBO}$ matrix elements then read:

$$
\begin{aligned}
\gamma_{k, l}\left(\Psi_{9}\right)= & \{1+\exp [\mathrm{i}(k-l) \pi / 3]\} / 3 \\
& +\{\exp [\mathrm{i}(l-k) \pi / 3]+\exp [\mathrm{i}(l-k) 2 \pi / 3]\} / 6 \text { and } \\
\gamma_{k, l}\left(\Psi_{10}\right)= & \{1+\exp [\mathrm{i}(k-l) \pi / 3]\} / 3 \\
& +\{\exp [\mathrm{i}(l-k) \pi / 3]+\exp [\mathrm{i}(k-l) 2 \pi / 3]\} / 6 .
\end{aligned}
$$


They accordingly predict

$$
\begin{gathered}
\gamma_{k, k}\left(\Psi_{9}\right)=1, \quad \gamma_{k, k+1}\left(\Psi_{9}\right)=1 / 2, \\
\gamma_{k, k+2}\left(\Psi_{9}\right)=-\mathrm{i} /(2 \sqrt{3}), \quad \gamma_{k, k+3}\left(\Psi_{9}\right)=0 \quad \text { and } \\
\gamma_{k, k}\left(\Psi_{10}\right)=1, \quad \gamma_{k, k+1}\left(\Psi_{10}\right)=1 / 2-\mathrm{i} /(2 \sqrt{3}), \\
\gamma_{k, k+2}\left(\Psi_{10}\right)=\gamma_{k, k+3}\left(\Psi_{10}\right)=0 .
\end{gathered}
$$

Similar complex CBO predictions follow from the associated pair of excitations from $\varphi_{1}^{*},\left(\varphi_{1}^{*} \rightarrow \varphi_{2}^{*}\right)$ and $\left(\varphi_{1}^{*} \rightarrow \varphi_{2}\right)$,

$$
\begin{aligned}
& \Psi_{11}=\operatorname{det}\left[\left(\pi_{1}^{+}, \pi_{1}^{-}\right),\left(\varphi_{1}^{+}, \varphi_{1}^{-}\right), \varphi_{1}^{*+}, \varphi_{2}^{*-}\right] \text { and } \\
& \Psi_{12}=\operatorname{det}\left[\left(\pi_{1}^{+}, \pi_{1}^{-}\right),\left(\varphi_{1}^{+}, \varphi_{1}^{-}\right), \varphi_{1}^{*+}, \varphi_{2}^{-}\right] .
\end{aligned}
$$

The relevant $\mathrm{CBO}$ data,

$$
\begin{aligned}
\gamma_{k, l}\left(\Psi_{11}\right)= & \{1+\exp [\mathrm{i}(l-k) \pi / 3]\} / 3 \\
& +\{\exp [\mathrm{i}(k-l) \pi / 3]+\exp [\mathrm{i}(k-l) 2 \pi / 3]\} / 6 \text { and } \\
\gamma_{k, l}\left(\Psi_{12}\right)= & \{1+\exp [\mathrm{i}(l-k) \pi / 3]\} / 3 \\
& +\{\exp [\mathrm{i}(k-l) \pi / 3]+\exp [\mathrm{i}(l-k) 2 \pi / 3]\} / 6,
\end{aligned}
$$

then predict:

$$
\begin{aligned}
& \gamma_{k, k}\left(\Psi_{11}\right)=1, \quad \gamma_{k, k+1}\left(\Psi_{11}\right)=1 / 2, \\
& \gamma_{k, k+2}\left(\Psi_{11}\right)=\mathrm{i} /(2 \sqrt{3}), \quad \gamma_{k, k+3}\left(\Psi_{11}\right)=0 \text { and } \\
& \gamma_{k, k}\left(\Psi_{12}\right)=1, \quad \gamma_{k, k+1}\left(\Psi_{12}\right)=1 / 2+\mathrm{i} /(2 \sqrt{3}), \\
& \gamma_{k, k+2}\left(\Psi_{12}\right)=\gamma_{k, k+3}\left(\Psi_{12}\right)=0 .
\end{aligned}
$$

One observes that CBO data for $\Psi_{11}$ and $\Psi_{12}$ represent the complex conjugates of the corresponding predictions for $\Psi_{9}$ and $\Psi_{10}$, respectively.

Consider now some energetically less accessible single excitations. The first pair represents the $\left(\pi_{1} \rightarrow \varphi_{2}\right)$ and $\left(\pi_{1} \rightarrow \varphi_{2}^{*}\right)$ configurations,

$$
\begin{aligned}
& \Psi_{13}=\operatorname{det}\left[\left(\varphi_{1}^{+}, \varphi_{1}^{-}\right),\left(\varphi_{1}^{*+}, \varphi_{1}^{*-}\right), \pi_{1}^{+}, \varphi_{2}^{-}\right] \text {and } \\
& \Psi_{14}=\operatorname{det}\left[\left(\varphi_{1}^{+}, \varphi_{1}^{-}\right),\left(\varphi_{1}^{*+}, \varphi_{1}^{*-}\right), \pi_{1}^{+}, \varphi_{2}^{*-}\right],
\end{aligned}
$$

for which general $\mathrm{CBO}$ expressions read:

$$
\begin{aligned}
& \gamma_{k, l}\left(\Psi_{13}\right)=(2 / 3) \cos [\pi(l-k) / 3]+(1 / 6)\{1+\exp [\mathrm{i}(l-k) 2 \pi / 3]\} \text { and } \\
& \gamma_{k, l}\left(\Psi_{14}\right)=(2 / 3) \cos [\pi(l-k) / 3]+(1 / 6)\{1+\exp [\mathrm{i}(k-l) 2 \pi / 3]\} .
\end{aligned}
$$


Again, they give rise to the mutually complex conjugate predictions:

$$
\begin{array}{ll}
\gamma_{k, k}\left(\Psi_{13}\right)=1, & \gamma_{k, k+1}\left(\Psi_{13}\right)=5 / 12+\mathrm{i} /(4 \sqrt{3}), \\
& \gamma_{k, k+2}\left(\Psi_{13}\right)=-1 / 4-\mathrm{i} /(4 \sqrt{3}), \quad \gamma_{k, k+3}\left(\Psi_{13}\right)=-1 / 3 \\
\gamma_{k, k}\left(\Psi_{14}\right)=1, & \gamma_{k, k+1}\left(\Psi_{14}\right)=5 / 12-\mathrm{i} /(4 \sqrt{3}), \\
& \gamma_{k, k+2}\left(\Psi_{14}\right)=-1 / 4+\mathrm{i} /(4 \sqrt{3}), \quad \gamma_{k, k+3}\left(\Psi_{14}\right)=-1 / 3 .
\end{array}
$$

The second conjugate pair involves the $\left(\varphi_{1} \rightarrow \pi_{6}\right)$ and $\left(\varphi_{1}^{*} \rightarrow \pi_{6}\right)$ excitations:

$$
\begin{aligned}
& \Psi_{15}=\operatorname{det}\left[\left(\pi_{1}^{+}, \pi_{1}^{-}\right),\left(\varphi_{1}^{*+}, \varphi_{1}^{*-}\right), \varphi_{1}^{+}, \pi_{6}^{-}\right] \text {and } \\
& \Psi_{16}=\operatorname{det}\left[\left(\pi_{1}^{+}, \pi_{1}^{-}\right),\left(\varphi_{1}^{+}, \varphi_{1}^{-}\right), \varphi_{1}^{*+}, \pi_{6}^{-}\right]
\end{aligned}
$$

for which:

$\gamma_{k, l}\left(\Psi_{15}\right)=(1 / 3)\left[1+\exp [\mathrm{i} \pi(k-l) / 3]+(1 / 6)\left\{(-1)^{k+l}+\exp [\mathrm{i}(l-k) \pi / 3]\right\}\right.$ and

$\gamma_{k, l}\left(\Psi_{16}\right)=(1 / 3)\left[1+\exp [\mathrm{i} \pi(l-k) / 3]+(1 / 6)\left\{(-1)^{k+l}+\exp [\mathrm{i}(k-l) \pi / 3]\right\}\right.$.

The specific predictions for $\pi$-electron populations and the ring or cross-ring bonds then read:

$$
\begin{array}{ll}
\gamma_{k, k}\left(\Psi_{15}\right)=1, & \gamma_{k, k+1}\left(\Psi_{15}\right)=5 / 12-\mathrm{i} /(4 \sqrt{3}), \\
\gamma_{k, k+2}\left(\Psi_{15}\right)=1 / 4-\mathrm{i} /(4 \sqrt{3}), \quad \gamma_{k, k+3}\left(\Psi_{15}\right)=-1 / 3 \\
\gamma_{k, k}\left(\Psi_{16}\right)=1, & \gamma_{k, k+1}\left(\Psi_{16}\right)=5 / 12+\mathrm{i} /(4 \sqrt{3}), \\
\gamma_{k, k+2}\left(\Psi_{16}\right)=1 / 4+\mathrm{i} /(4 \sqrt{3}), \quad \gamma_{k, k+3}\left(\Psi_{16}\right)=-1 / 3 .
\end{array}
$$

Finally, we examine two pairs of the conjugate configurations resulting from the localized double excitations from $\left(\varphi_{1}, \varphi_{1}^{*}\right)$ to $\left(\varphi_{2}, \varphi_{2}^{*}\right) \mathrm{MO}$ :

$$
\begin{aligned}
& \Psi_{17}=\operatorname{det}\left[\left(\pi_{1}^{+}, \pi_{1}^{-}\right),\left(\varphi_{1}^{*+}, \varphi_{1}^{*-}\right),\left(\varphi_{2}^{+}, \varphi_{2}^{-}\right)\right], \\
& \Psi_{18}=\operatorname{det}\left[\left(\pi_{1}^{+}, \pi_{1}^{-}\right),\left(\varphi_{1}^{+}, \varphi_{1}^{-}\right),\left(\varphi_{2}^{*+}, \varphi_{2}^{*-}\right)\right],
\end{aligned}
$$

and

$$
\begin{aligned}
& \Psi_{19}=\operatorname{det}\left[\left(\pi_{1}^{+}, \pi_{1}^{-}\right),\left(\varphi_{1}^{*+}, \varphi_{1}^{*-}\right),\left(\varphi_{2}^{*+}, \varphi_{2}^{*-}\right)\right], \\
& \Psi_{20}=\operatorname{det}\left[\left(\pi_{1}^{+}, \pi_{1}^{-}\right),\left(\varphi_{1}^{+}, \varphi_{1}^{-}\right),\left(\varphi_{2}^{+}, \varphi_{2}^{-}\right)\right] .
\end{aligned}
$$

General expressions for the $\mathrm{CBO}$ data in $\mathrm{AO}$ resolution then read:

$$
\begin{aligned}
& \gamma_{k, l}\left(\Psi_{17}\right)=(1 / 3)[1+\exp [\mathrm{i} \pi(k-l) / 3]+\exp [\mathrm{i} 2(l-k) \pi / 3]], \\
& \gamma_{k, l}\left(\Psi_{18}\right)=(1 / 3)[1+\exp [\mathrm{i} \pi(l-k) / 3]+\exp [\mathrm{i} 2(k-l) \pi / 3]],
\end{aligned}
$$




$$
\begin{aligned}
& \gamma_{k, l}\left(\Psi_{19}\right)=(1 / 3)[1+\exp [\mathrm{i} \pi(k-l) / 3]+\exp [\mathrm{i} 2(k-l) \pi / 3]], \\
& \gamma_{k, l}\left(\Psi_{20}\right)=(1 / 3)[1+\exp [\mathrm{i} \pi(l-k) / 3]+\exp [\mathrm{i} 2(l-k) \pi / 3]] .
\end{aligned}
$$

They give rise to the following atomic populations and the ring inter-carbon bond orders of $\pi$-electrons:

$$
\begin{aligned}
& \gamma_{k, k}\left(\Psi_{17}\right)=1, \quad \gamma_{k, k+1}\left(\Psi_{17}\right)=1 / 3, \quad \gamma_{k, k+2}\left(\Psi_{17}\right)=-\mathrm{i} / \sqrt{3}, \quad \gamma_{k, k+3}\left(\Psi_{17}\right)=1 / 3 \\
& \gamma_{k, k}\left(\Psi_{18}\right)=1, \quad \gamma_{k, k+1}\left(\Psi_{18}\right)=1 / 3, \quad \gamma_{k, k+2}\left(\Psi_{18}\right)=\mathrm{i} / \sqrt{3}, \quad \gamma_{k, k+3}\left(\Psi_{18}\right)=1 / 3 \\
& \gamma_{k, k}\left(\Psi_{19}\right)=1, \quad \gamma_{k, k+1}\left(\Psi_{19}\right)=1 / 3-\mathrm{i} / \sqrt{3}, \quad \gamma_{k, k+2}\left(\Psi_{19}\right)=0, \quad \gamma_{k, k+3}\left(\Psi_{19}\right)=1 / 3 \\
& \gamma_{k, k}\left(\Psi_{20}\right)=1, \quad \gamma_{k, k+1}\left(\Psi_{20}\right)=1 / 3+\mathrm{i} / \sqrt{3}, \quad \gamma_{k, k+2}\left(\Psi_{20}\right)=0, \quad \gamma_{k, k+3}\left(\Psi_{20}\right)=1 / 3
\end{aligned}
$$

In each pair of these electronic states the predicted values of the corresponding bond orders are again seen to be complex conjugates of each other.

\section{Bond descriptors and information measures in complex states}

In all configurations the predicted electron populations of $\pi$-electron basis functions are equal, $\gamma_{k, k}=1$, for all carbons in the ring. The predicted bond-orders $(k \neq l)$

$$
\gamma_{k, l}=\operatorname{Re}\left(\gamma_{k, l}\right)+\mathrm{i} \operatorname{Im}\left(\gamma_{k, l}\right)=M_{k, l} \exp \left(\mathrm{i} \phi_{k, l}\right)
$$

in the selected $\pi$-electronic states of benzene are listed in Table 1, together with their modulus $\left(M_{k, l}\right)$ and phase ( $\phi_{k, l}$, in degrees) characteristics (see also Fig. 1):

$$
M_{k, l}=\left\{\left[\operatorname{Re}\left(\gamma_{k, l}\right)\right]^{2}+\left[\operatorname{Im}\left(\gamma_{k, l}\right)\right]^{2}\right\}^{1 / 2}, \quad \phi_{k, l}=\arctan \left[\operatorname{Im}\left(\gamma_{k, l}\right) / \operatorname{Re}\left(\gamma_{k, l}\right)\right]
$$

One observes in the table that the para bond-orders are real for all configurations considered, thus giving rise to the vanishing contributions from these cross ring bonds to the resultant electronic current.

As argued elsewhere [33-43] the chemical intuition on bond multiplicities $\left\{N_{k, l}(\alpha)\right\}$ in the given configuration $\Psi_{\alpha}$ is well reflected by the squares of the real CBO matrix elements $\left\{\gamma_{k, l}(\alpha)\right\}$,

$$
N_{k, l}(\alpha)=\operatorname{sign}\left[\gamma_{k, l}(\alpha)\right]\left[\gamma_{k, l}(\alpha)\right]^{2}
$$

with their signs keeping the memory of the bonding (plus) or antibonding (minus) character of the underlying AO interactions. The complex generalization of this Wibergtype approach calls for the chemical bond-order measure determined solely by the 
Table 1 The bond orders for the ortho, meta and para $\pi$-bonds of benzene carbon ring in selected electron configurations

\begin{tabular}{|c|c|c|c|c|c|c|c|c|c|}
\hline \multirow[t]{2}{*}{$\alpha$} & \multicolumn{3}{|l|}{ Ortho } & \multicolumn{3}{|l|}{ Meta } & \multicolumn{3}{|l|}{ Para } \\
\hline & $\gamma_{k, k+1}$ & $M_{k, k+1}$ & $\phi_{k, k+1}$ & $\gamma_{k, k+2}$ & $M_{k, k+2}$ & $\phi_{k, k+2}$ & $\gamma_{k, k+3}$ & $M_{k, k+3}$ & $\phi_{k, k+3}$ \\
\hline 0 & $2 / 3$ & $2 / 3$ & 0 & 0 & 0 & 0 & $-1 / 3$ & $1 / 3$ & 0 \\
\hline 1 & $1 / 3$ & $1 / 3$ & 0 & 0 & 0 & 0 & $1 / 3$ & $1 / 3$ & 0 \\
\hline 2 & $-2 / 3$ & $2 / 3$ & 0 & 0 & 0 & 0 & $1 / 3$ & $1 / 3$ & 0 \\
\hline 3 & 0 & 0 & 0 & 0 & 0 & 0 & 1 & 1 & 0 \\
\hline 4 & 0 & 0 & 0 & 0 & 0 & 0 & $-1 / 3$ & $1 / 3$ & 0 \\
\hline 5 & $1 / 3$ & $1 / 3$ & 0 & 0 & 0 & 0 & $-2 / 3$ & $2 / 3$ & 0 \\
\hline 6 & $-1 / 3$ & $1 / 3$ & 0 & 0 & 0 & 0 & $2 / 3$ & $2 / 3$ & 0 \\
\hline 7 & 0 & 0 & 0 & 0 & 0 & 0 & 0 & 0 & 0 \\
\hline 8 & $-1 / 3$ & $1 / 3$ & 0 & 0 & 0 & 0 & $-1 / 3$ & $1 / 3$ & 0 \\
\hline 9 & $1 / 2$ & $1 / 2$ & 0 & $-\mathrm{i} /(2 \sqrt{3})$ & $1 / \sqrt{12}$ & 90 & 0 & 0 & 0 \\
\hline 10 & $1 / 2-i /(2 \sqrt{3})$ & $1 / \sqrt{3}$ & -30 & 0 & 0 & 0 & 0 & 0 & 0 \\
\hline 11 & $1 / 2$ & $1 / 2$ & 0 & $\mathrm{i} /(2 \sqrt{3})$ & $1 / \sqrt{12}$ & 90 & 0 & 0 & 0 \\
\hline 12 & $1 / 2+i /(2 \sqrt{3})$ & $1 / \sqrt{3}$ & 30 & 0 & 0 & 0 & 0 & 0 & 0 \\
\hline 13 & $5 / 12+i /(4 \sqrt{3})$ & $\sqrt{7} / 6$ & 19 & $-1 / 4-i /(4 \sqrt{3})$ & $1 / \sqrt{12}$ & 30 & $-1 / 3$ & $1 / 3$ & 0 \\
\hline 14 & $5 / 12-\mathrm{i} /(4 \sqrt{3})$ & $\sqrt{7} / 6$ & -19 & $-1 / 4+i /(4 \sqrt{3})$ & $1 / \sqrt{12}$ & -30 & $-1 / 3$ & $1 / 3$ & 0 \\
\hline 15 & $5 / 12-\mathrm{i} /(4 \sqrt{3})$ & $\sqrt{7} / 6$ & -19 & $1 / 4-i /(4 \sqrt{3})$ & $1 / \sqrt{12}$ & -30 & $-1 / 3$ & $1 / 3$ & 0 \\
\hline 16 & $5 / 12+\mathrm{i} /(4 \sqrt{3})$ & $\sqrt{7} / 6$ & 19 & $1 / 4+\mathrm{i} /(4 \sqrt{3})$ & $1 / \sqrt{12}$ & 30 & $-1 / 3$ & $1 / 3$ & 0 \\
\hline 17 & $1 / 3$ & $1 / 3$ & 0 & $-\mathrm{i} / \sqrt{3}$ & $1 / \sqrt{3}$ & -30 & $1 / 3$ & $1 / 3$ & 0 \\
\hline 18 & $1 / 3$ & $1 / 3$ & 0 & $i / \sqrt{3}$ & $1 / \sqrt{3}$ & 30 & $1 / 3$ & $1 / 3$ & 0 \\
\hline 19 & $1 / 3-i / \sqrt{3}$ & $2 / 3$ & -60 & 0 & 0 & 0 & $1 / 3$ & $1 / 3$ & 0 \\
\hline 20 & $1 / 3+i / \sqrt{3}$ & $2 / 3$ & 60 & 0 & 0 & 0 & $1 / 3$ & $1 / 3$ & 0 \\
\hline
\end{tabular}

Fig. 1 The complex CBO matrix element $\gamma_{k, l}=M_{k, l} \exp \left(\mathrm{i} \phi_{k, l}\right)$ and its use in diagnosing the bonding status of the chemical $\pi$ interactions between carbons $k$ and $l$ in the benzene ring

\section{Nonbonding: $\phi_{k, l}=\pi / 2$}

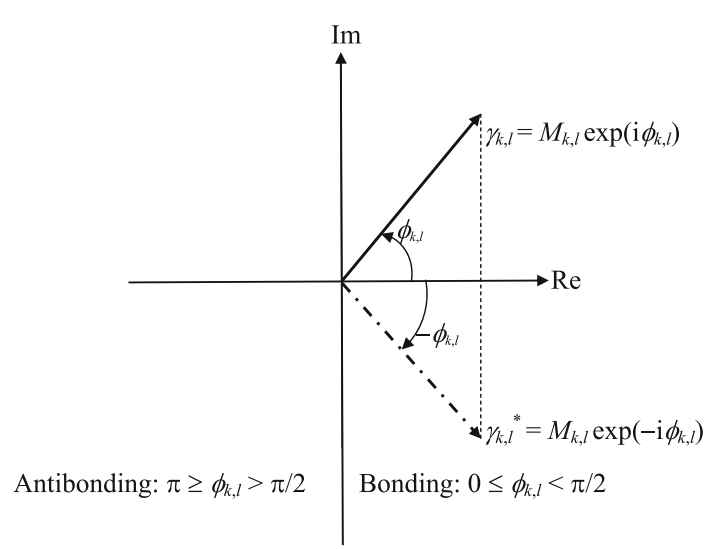


real part $\operatorname{Re}\left[\gamma_{k, l}(\alpha)\right]$ of the complex matrix element:

$$
N_{k, l}(\alpha)=\operatorname{sign}\left[\operatorname{Re}\left(\gamma_{k, l}\right)\right]\left\{\operatorname{Re}\left[\gamma_{k, l}(\alpha)\right]\right\}^{2} .
$$

Of interst also are some global bond-multiplicity indices, of the system as a whole, e.g., the overall numbers of the bonding ( $b$, positive) and antibonding ( $a$, negative) inter-atomic interactions in $\Psi_{\alpha}$,

$$
\begin{aligned}
& N_{b}(\alpha)=\sum \sum_{k<l} N_{k, l}(\alpha), \quad N_{k, l}(\alpha)>0, \\
& N_{a}(\alpha)=\sum \sum_{k<l} N_{k, l}(\alpha), \quad N_{k, l}(\alpha)<0,
\end{aligned}
$$

and their sum measuring the resultant global multiplicity:

$$
\Delta N(\alpha)=N_{b}(\alpha)+N_{a}(\alpha)
$$

Next, let us examine expressions for the nonclassical information contributions in this single-determinant approximation of the wave function describing the ring $\pi$ electrons, $\Psi(\pi)=\operatorname{det}(\varphi)$, expressed in terms of the configuration occupied $\pi$-MO

$$
\boldsymbol{\varphi}(\boldsymbol{r})=\left\{\varphi_{S}(\boldsymbol{r}) \equiv R_{S}(\boldsymbol{r}) \exp \left[\mathrm{i} \Phi_{S}(\boldsymbol{r})\right]\right\},
$$

each expanded in the real AO basis $\chi(\boldsymbol{r})=\left\{\chi_{k}(\boldsymbol{r})\right\}$ of the six carbon $2 p_{z}$ orbitals,

$$
\varphi(\boldsymbol{r})=\chi(\boldsymbol{r}) \mathbf{Z}
$$

The LCAO MO coefficients $\mathbf{Z}$ are generally complex,

$$
\mathbf{Z}=\left\{Z_{k, s}=M_{k, s} \exp \left(\mathrm{i} \phi_{k, s}\right)\right\}
$$

being characterized by their respective moduli $\left\{M_{k, s}\right\}$ and phases $\left\{\phi_{k, s}\right\}$ in the complex plane. The resultant MO phase $\left[\Phi_{S}(\boldsymbol{r})\right]$ and modulus $\left[R_{S}(\boldsymbol{r})\right]$ functions of the representative $\mathrm{MO}$

$$
\varphi_{s}(\boldsymbol{r})=\Sigma_{k}\left[\chi_{k}(\boldsymbol{r}) M_{k, s}\right] \exp \left(\mathrm{i} \phi_{k, s}\right) \equiv \Sigma_{k} m_{k, s}(\boldsymbol{r}) \exp \left(\mathrm{i} \phi_{k, s}\right) \equiv R_{s}(\boldsymbol{r}) \exp \left[\mathrm{i} \Phi_{s}(\boldsymbol{r})\right]
$$

then read:

$$
\begin{aligned}
\Phi_{s}(\boldsymbol{r}) & =\arctan \left\{\left[\Sigma_{k} m_{k, s}(\boldsymbol{r}) \sin \left(\phi_{k, s}\right)\right] /\left[\Sigma_{k} m_{k, s}(\boldsymbol{r}) \cos \left(\phi_{k, s}\right)\right]\right\} \\
& =\arctan \left\{\operatorname{Im}\left[\varphi_{s}(\boldsymbol{r})\right] / \operatorname{Re}\left[\varphi_{s}(\boldsymbol{r})\right]\right\} \\
R_{S}(\boldsymbol{r}) & =\left\{\operatorname{Re}\left[\varphi_{s}(\boldsymbol{r})\right]^{2}+\operatorname{Im}\left[\varphi_{s}(\boldsymbol{r})\right]^{2}\right\}^{1 / 2}
\end{aligned}
$$


The configuration (phase/current)-related entropy/information quantities can be then expressed as sums of their expectation values in the occupied MO states:

$$
S^{\text {nclass. }}[\Psi]=\Sigma_{s} n_{s} S^{\text {nclass. }}\left[\varphi_{s}\right] \text { and } I^{\text {nclass. }}[\Psi]=\Sigma_{s} n_{s} I^{\text {nclass. }}\left[\varphi_{s}\right]
$$

here $n_{s}$ is the MO occupation in configuration $\Psi_{0}(\pi)$ and the MO expectation values can be expressed in terms of the MO resultant modulus and phase functions:

$$
\begin{aligned}
S^{\text {nclass. }\left[\varphi_{s}\right]} & =\left\langle\varphi_{s}\left|\hat{\mathrm{S}}^{\text {nclass. }}\right| \varphi_{s}\right\rangle=-2 \int p_{s}(\boldsymbol{r})\left|\Phi_{s}(\boldsymbol{r})\right| d \boldsymbol{r} \\
& \equiv-2 \int p_{s}(\boldsymbol{r}) \Phi_{S}(\boldsymbol{r}) d \boldsymbol{r}, \quad p_{s}(\boldsymbol{r})=\left[R_{S}(\boldsymbol{r})\right]^{2} ; \\
I^{\text {nclass. }}\left[\varphi_{s}\right] & =\left\langle\varphi_{S}\left|\hat{\mathrm{I}}^{\text {nclass. }}\right| \varphi_{S}\right\rangle=4 \int p_{S}(\boldsymbol{r})\left[\nabla \Phi_{S}(\boldsymbol{r})\right]^{2} d \boldsymbol{r} .
\end{aligned}
$$

The overall average measures are then obtained as integrals over local traces:

$$
\begin{aligned}
& S^{\text {nclass. }}[\Psi]=-2 \int \operatorname{tr}[\mathbf{F}(\boldsymbol{r} ; s) \boldsymbol{\omega}(\boldsymbol{r})] d \boldsymbol{r}, \\
\mathbf{F}(\boldsymbol{r} ; s)= & \left\{F_{k, l}(\boldsymbol{r} ; s)=\Sigma_{s} Z_{k, s}^{*}\left[n_{s} \Phi_{s}(\boldsymbol{r})\right] Z_{l, s}\right\}, \\
& I^{\text {nclass. }}[\Psi]=4 \int \operatorname{tr}[\mathbf{G}(\boldsymbol{r} ; s) \boldsymbol{\omega}(\boldsymbol{r})] d \boldsymbol{r}, \\
\mathbf{G}(\boldsymbol{r} ; s)= & \left\{G_{l, k}(\boldsymbol{r} ; s)=\Sigma_{s} Z_{k, s}^{*}\left\{n_{s}\left[\nabla \Phi_{s}(\boldsymbol{r})\right]^{2}\right\} Z_{l, s}\right\} .
\end{aligned}
$$

These nonclassical entropy and gradient (Fisher-type) information functionals are thus generated by the functional densities determined by traces of products of the local matrices of the phase-magnitude, $\mathbf{F}(\boldsymbol{r} ; s)$, or the phase-gradient, $\mathbf{G}(\boldsymbol{r} ; s)$, and the AO overlap matrix $\boldsymbol{\omega}(\boldsymbol{r})=\left\{\omega_{k, l}(\boldsymbol{r})=\chi_{k}(\boldsymbol{r}) \chi_{l}(\boldsymbol{r})\right\}$ groups the corresponding AO products.

The electron density $\rho_{\alpha}(\boldsymbol{r})$ of $\pi$-electrons in the configuaration $\Psi \alpha=\operatorname{det}\left(\boldsymbol{\varphi}_{\alpha}\right)$ can be similarly expressed in terms of MO densities $\left\{\rho_{s}(\boldsymbol{r})\right\}$ or the associated probability distributions $\left\{p_{s}(\boldsymbol{r})=\rho_{s}(\boldsymbol{r}) / n_{s}\right\}$ of the state occupied $\mathrm{MO} \boldsymbol{\varphi}_{\alpha}$ :

$$
\begin{aligned}
\rho_{\alpha}(\boldsymbol{r}) & =\sum_{s}^{o c c d(\alpha)} n_{s} \varphi_{s}(\boldsymbol{r}) \varphi_{s}^{*}(\boldsymbol{r}) \equiv \sum_{s}^{o c c d(\alpha)} n_{s} p_{s}(\boldsymbol{r}) \equiv \sum_{s}^{o c c d(\alpha)} \rho_{s}(\boldsymbol{r}) \\
& =\Sigma_{k} \Sigma_{l}\left(\Sigma_{s} Z_{k, s} n_{s} Z_{l, s}^{*}\right) \chi_{l}(\boldsymbol{r}) \chi_{k}(\boldsymbol{r}) \equiv \Sigma_{k} \Sigma_{l} \gamma_{l, k} \omega_{k, l}(\boldsymbol{r}) \equiv \operatorname{tr}[\boldsymbol{\gamma} \boldsymbol{\omega}(\boldsymbol{r})] .
\end{aligned}
$$

The corresponding expression for the average current, which explores the gradient of the MO phases, involves only the imaginary part of the $\mathrm{CBO}$ matrix, $\operatorname{Im}(\boldsymbol{\gamma})=$ $1 /(2 \mathrm{i})\left(\boldsymbol{\gamma}-\boldsymbol{\gamma}^{*}\right)$. Its real part, $\operatorname{Re}(\boldsymbol{\gamma})=(1 / 2)\left(\boldsymbol{\gamma}+\boldsymbol{\gamma}^{*}\right)$, similarly determines a pattern of the chemical bond multiplicities in the electronic state under consideration (see Fig. 1 and Table 2).

Indeed, in the preceding section we have already used the sign of the (real) CBO matrix elements, of the symmetric-population configurations $\left(\Psi_{0}, \ldots, \Psi_{8}\right)$, in a qual- 
Table 2 Orbital excitation energies and $\pi$ bond multiplicitis in benzene

\begin{tabular}{lllllllll}
\hline$\alpha$ & $\Delta E$ & $N_{k, k+1}$ & $N_{k, k+2}$ & $N_{k, k+3}$ & $N_{b}$ & $-N_{a}$ & $\Delta N$ & $\Delta N_{\text {ortho }}$ \\
\hline 0 & 0 & $4 / 9$ & 0 & $-1 / 9$ & $8 / 3$ & $1 / 3$ & $7 / 3$ & $8 / 3$ \\
1 & 4 & $1 / 9$ & 0 & $1 / 9$ & 1 & 0 & 1 & $2 / 3$ \\
2 & 16 & $-4 / 9$ & 0 & $1 / 9$ & $1 / 3$ & $8 / 3$ & $-7 / 3$ & $-8 / 3$ \\
3 & 8 & 0 & 0 & 1 & 3 & 0 & 3 & 0 \\
4 & 8 & 0 & 0 & $-1 / 9$ & 0 & $1 / 3$ & $-1 / 3$ & 0 \\
5 & 4 & $1 / 9$ & 0 & $-4 / 9$ & $2 / 3$ & $4 / 3$ & $-2 / 3$ & $2 / 3$ \\
6 & 12 & $-1 / 9$ & 0 & $4 / 9$ & $4 / 3$ & $2 / 3$ & $2 / 3$ & $-2 / 3$ \\
7 & 8 & 0 & 0 & 0 & 0 & 0 & 0 & 0 \\
8 & 12 & $-1 / 9$ & 0 & $-1 / 9$ & 0 & 1 & -1 & $-2 / 3$ \\
$9 \div 12$ & 2 & $1 / 4$ & 0 & 0 & $3 / 2$ & 0 & $3 / 2$ & $3 / 2$ \\
13,14 & 3 & $25 / 144$ & $-1 / 16$ & $-1 / 9$ & $25 / 24$ & $17 / 24$ & $1 / 3$ & $25 / 24$ \\
15,16 & 3 & $25 / 144$ & $1 / 16$ & $-1 / 9$ & $17 / 12$ & $1 / 3$ & $13 / 12$ & $25 / 24$ \\
$17 \div 20$ & 4 & $1 / 9$ & 0 & $1 / 9$ & 1 & 0 & 1 & $2 / 3$ \\
\hline
\end{tabular}

itative diagnosis of the bonding $\left(\gamma_{k, l}>0\right)$, nonbonding $\left(\gamma_{k, l}=0\right)$, and antibonding $\left(\gamma_{k, l}<0\right)$ character of the inter-atomic chemical interactions. The complex-plane generalization of this criterion for $\gamma_{k, l}=M_{k, l} \exp \left(\mathrm{i} \phi_{k, l}\right)$, in terms of the the $\gamma_{k, l}$-phase, $\phi_{k, l}$, is shown in Fig. 1: the left half-plane $\left(\pi \geq \phi_{k, l}>\pi / 2\right)$ covers the antibonding interactions, the imaginary axis $\left(\phi_{k, l}=\pi / 2\right)$ corresponds to the nonbonding character, while te right half-plane $\left(0 \leq \phi_{k, l}<\pi / 2\right)$ marks the chemically $\pi$-bonded status of the specified pair of carbon atoms.

A chemical relevance of the $\mathrm{CBO}$ matrix elements and the bond multiplicities they imply is also revealed by their dependencies on the $\pi$-electron orbital excitation energy reative to the ground-state. The accepted chemical intuition indeed suggests that with increasing excitation energy, given by the difference of the $\pi$-MO energies $\left\{\varepsilon_{s}\right\}$ occupied in $\Psi_{\alpha}$ and $\Psi_{0}$, respectively,

$$
\Delta E(\alpha)=\sum_{s}^{o c c d(\alpha)} n_{s} \varepsilon_{s}-\sum_{s}^{o c c d(0)} n_{s} \varepsilon_{s} \equiv E(\alpha)-E(0),
$$

there should be less overall bonding $\left[N_{b}(\alpha)\right]$ and more antibonding $\left[-N_{a}(\alpha)\right]$ bond multiplicity descriptors in the $\pi$ system. One is also interested how this energy influences individual matrix elements corresponding to the ortho, meta and para interactions in benzene. Thus, a net excess $\Delta N(\alpha)$ of the bonding (positive) interactions over antibonding (negative) ones, is also expected to exhibit a decreasing (nonlinear) correlation with increasing $\Delta E(\alpha)$. One recalls that this energy difference also determines the amount of mixing of the excited configuration $\Psi_{\alpha}$ into the (correlated) ground state in the Configuration Interaction $(\mathrm{CI})$ representation,

$$
\Psi_{0}^{\mathrm{CI}}=\Sigma_{\alpha} \Psi_{\alpha} C_{\alpha, 0},
$$


Fig. 2 The

$\operatorname{Re}\left[\gamma_{k, l}(\alpha)\right]=\operatorname{Re}\left[\gamma_{k, l}(\Delta E(\alpha)]\right.$ plots for the ortho $(l=k+1, \mathbf{a})$, meta $(l=k+2, \mathbf{b})$ and para $(l=k+3, \mathbf{c}) \pi$-bonds in benzene

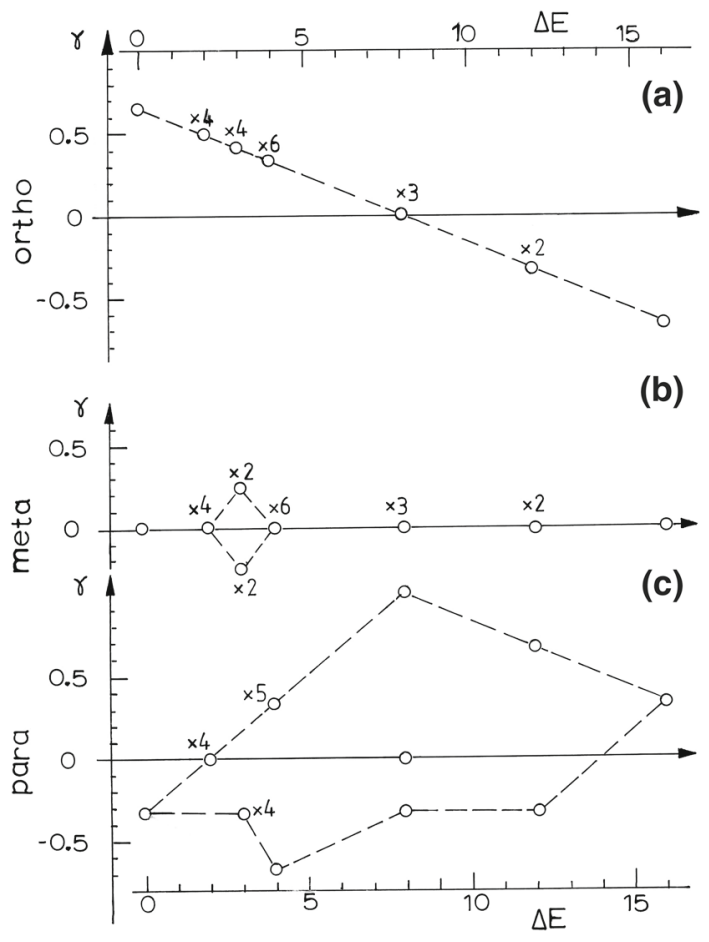

with the perturbational expansion coefficient $C_{\alpha, 0}=\left\langle\Psi_{\alpha} \mid \Psi_{0}^{\mathrm{CI}}\right\rangle$ being inversely proportional to $\Delta E(\alpha)$.

One should also recognize the known differences between the ring (ortho, strongoverlap) and cross-ring (meta, para, weak-overlap) interactions, with the former dominating the overall chemical bond stabilization of the benzene $\pi$-bond system. Therefore, one would expect similar correlation between increasing $\Delta E(\alpha)$ and the resultant bond multiplicity index limited only to the strongest (nearest-neighbor) ortho bonds:

$$
\begin{aligned}
\Delta N_{\text {ortho }}(\alpha)= & \left\{N_{b}(\alpha, \text { ortho })=6 N_{\text {ortho }}(\alpha), \text { for } \gamma_{\text {ortho }}(\alpha)>0 ;\right. \\
& \left.N_{a}(\alpha, \text { ortho })=6 N_{\text {ortho }}(\alpha), \text { for } \gamma_{\text {ortho }}(\alpha)<0\right\} .
\end{aligned}
$$

One observes that there are altogether 6 distinct ortho and meta bonds, and three para interactions in benzene ring, each exhibiting the same bond-order or bond-multiplicity characteristics.

The relevant excitation energies, in units $(-\beta)>0$, and bond multiplicities, generated using the $\mathrm{CBO}$ data grouped in Table 1, are listed in Table 2, while selected correlations with respect to the MO excitation energy are tested in Figs. 2 and 3. The aim of the former figure is to check energy correlations of the real part of the $\pi$ bondorders themselves, while the latter investigates the energetical dependence of their squares, i.e., the multiplicities of the ortho, meta and para $\pi$ bonds in the benzene ring. In the first $\Delta N(\alpha)=\Delta N[\Delta E(\alpha)]$ plot of Fig. 3, predictions for $\Psi_{3}, \Psi_{5}$ and $\Psi_{6}$, which do not affect the second $\Delta N_{\text {ortho }}(\alpha)=\Delta N_{\text {ortho }}[\Delta E(\alpha)]$ plot o the figure, have 
Fig. 3 The

$\Delta N(\alpha)=\Delta N[\Delta E(\alpha)]$ (a) and

$\Delta N_{\text {ortho }}(\alpha)=$

$\Delta N_{\text {ortho }}[\Delta E(\alpha)](\mathbf{b})$ plots for

$\pi$-bonds in benzene
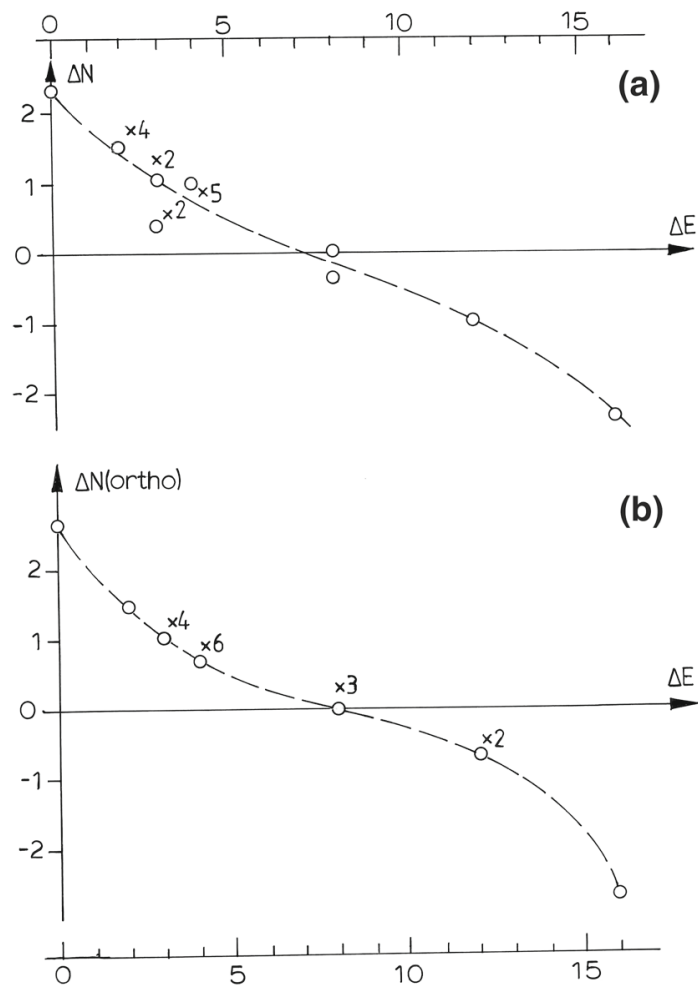

been ignored as dominated by multiplicities of the weakest para-interactions (see also Table 2).

A reference to Tables 1 and 2 and Figs. 2 and 3 shows that the crucial ortho-bonds indeed exhibit a decreasing correlations $\gamma_{k, k+1}(\Delta E)$ and $\Delta N_{\text {ortho }}(\Delta E)$ of the bondorder/multiplicity measures with increasing excitation energy. This is clearly seen in the exactly linear CBO correlation of Fig. 2a and the associated bond multiplicity plot of Fig. 3b. The para interactions, always marked by the real bond-orders, are seen to generate a scatter of points in a wide area of the CBO plot shown in Fig. 2c. It is responsible for a lower quality of the overall multiplicity correlation (Fig. 3a) relative to the ortho-only plot (Fig. 3b). The predicted meta bond orders stay practically nonbonded, thus giving rise to a vanishing bond multiplicity and remaining unaffected by electron excitations. This analysis indicates that the strong ortho-bonds alone should be recommended in probing changes of the chemical bond pattern upon excitation. The erratic, small influence of the cross-ring bonds only distorts predictions, compared to chemical expectations.

One also observes that the ground-state configuration $\Psi_{0}$ does not generate the highest multiplicity of all $\pi$-bonds in the system. This maximum value is reached in the excited configuration $\Psi_{3}$, giving rise to exactly three (weakest) para-bonds in the benzene ring. Therefore, the "entropic" quantity of the overall bond multiplicity of all bonds should not be associated with the chemical bond-strength or bond-energy concepts. The latter are more closely linked, though, to the overall multiplicity of 
the strongest (ortho) bonds in the benzene ring, with the weak cross-ring interactions being either energetically irrelevant (meta) or contributing only a small, "noisy" supplement (para) to the resultant bond-stabilization energy in these excited electron configurations.

\section{Conclusion}

In degenerate electronic states the AO density matrix can involve the complex elements leading to a nonvanishing spatial phase of the system wave function and hence a finite probability current. This gives rise to non-zero nonclassical (phase/current related) entropy and information descriptors and calls for a generalization of the multiplicity descriptors used to explore the state bond pattern. In this work we have summarized the quantum supplements of the classical entropy/information concepts, to accommodate such complex wave functions (probability amplitudes) of the quantum-mechanical description of molecular electronic states. The phase and resultant entropy continuity problems have also been reexamined. In this phenomenological framework, which results directly from the Schrödinger equation, one introduces the concept of the entropy-current and identifies the associated entropy-production term. This approach can be applied to determine rates of the equilibrium molecular relaxations and their characteristic time descriptors. An illustrative example of the transition from the promolecule (non-stationary system consisting of the molecularly placed "frozen" atomic distributions) to the molecule (stationary ground-state system) has been selected. The temporal aspect of the promolecule $\rightarrow$ molecule structure reorganization has been qualitatively examined in $\mathrm{H}_{2}^{+}$.

The excited configurations of the $\pi$-electron system in benzene ring have been explored using the (ground-state) equivalent, complex MO framework in the Hückel theory. The configuration bond-orders for the ring (ortho) and cross-ring (meta, para) interactions have been determined and correlated with the orbital excitation energy $\Delta E$. With the increasing energy difference the ortho interactions decrease linearly, meta bond orders remain insensitive (nonbonding), while para matrix elements behave "chaotically". This suggests using just the nearest-neighbor (ortho) indices as the most adequate indicators of a changing bond pattern with electronic excitations. The Wiberg-type bond-multiplicity concept has been extended to cover the complex CBO matrix elements with the chemically relevant index being determined by their real part alone. These bond descriptors also display a decreasing trend with respect to the excitation energy, thus exhibiting less bonding (more antibonding) character with increasing relative energy of the excited configurations.

Open Access This article is distributed under the terms of the Creative Commons Attribution License which permits any use, distribution, and reproduction in any medium, provided the original author(s) and the source are credited.

\section{References}

1. R.A. Fisher, Proc. Camb. Philos. Soc. 22, 700 (1925) 
2. B.R. Frieden, Physics from the Fisher Information: A Unification, 2nd edn. (Cambridge University Press, Cambridge, 2004)

3. C.E. Shannon, Bell Syst. Tech. J. 27, 379, 623 (1948)

4. C.E. Shannon, W. Weaver, The Mathematical Theory of Communication (University of Illinois, Urbana, 1949)

5. S. Kullback, R.A. Leibler, Ann. Math. Stat. 22, 79 (1951)

6. S. Kullback, Information Theory and Statistics (Wiley, New York, 1959)

7. N. Abramson, Information Theory and Coding (McGraw-Hill, New York, 1963)

8. P.E. Pfeifer, Concepts of Probability Theory, 2nd edn. (Dover, New York, 1978)

9. R.F. Nalewajski, J. Math. Chem. 51, 297 (2013)

10. R.F. Nalewajski, Found. Chem. 16, 27 (2014)

11. R.F. Nalewajski, Information Theory of Molecular Systems (Elsevier, Amsterdam, 2006)

12. R.F. Nalewajski, Information Origins of the Chemical Bond (Nova, New York, 2010)

13. R.F. Nalewajski, Perspectives in Electronic Structure Theory (Springer, Heidelberg, 2012)

14. R.F. Nalewajski, in Mathematical Chemistry, ed. by W.I. Hong (Nova Science, New York, 2011), pp. 247-325

15. R.F. Nalewajski, in Chemical Information and Computation Challenges, in 21st Century, ed. by M.V. Putz (Nova Science, New York, 2012), pp. 61-100

16. R.F. Nalewajski, P. de Silva, J. Mrozek, in Theoretical and Computational Developments in Modern Density Functional Theory, ed. by A.K. Roy (Nova Science, New York, 2012), pp. 561-588

17. R.F. Nalewajski, in Frontiers in Modern Theoretical Chemistry: Concepts and Methods (Dedicated to B. M. Deb), ed. by P.K. Chattaraj, S.K. Ghosh (Taylor \& Francis/CRC, London, 2013), pp. 143-180

18. R.F. Nalewajski, in Applications of Density Functional Theory to Chemical Reactivity, Struct. Bond. 149, 51 (2012)

19. R.F. Nalewajski, J. Phys. Chem A 107, 3792 (2003)

20. R.F. Nalewajski, Ann. Phys. (Leipzig) 13, 201 (2004)

21. R.F. Nalewajski, Mol. Phys. 112, 2587 (2014)

22. R.F. Nalewajski, J. Math. Chem. 51, 369 (2013)

23. R.F. Nalewajski, Ann. Phys. (Leipzig) 525, 256 (2013)

24. R.F. Nalewajski, J. Math. Chem. 52, 588 (2014)

25. R.F. Nalewajski, J. Math. Chem. 52, 1921 (2014)

26. R.F. Nalewajski, J. Math. Chem. (2014). doi:10.1007/s10910-014-0405-2

27. R.F. Nalewajski, in Advances in Quantum Systems Research, ed. by Z. Ezziane (Nova, New York, 2014), pp. 119-167

28. R.F. Nalewajski, Int. J. Quantum Chem. (2014). doi:10.1002/qua.24750

29. R.F. Nalewajski, Eur. Phys. J. D (2014, submitted)

30. R.F. Nalewajski, Advances in Mathematics Research, ed. by A.R. Baswell (Nova Science, New York, 2014, in press)

31. R.F. Nalewajski, Quantum Matter 4, 12 (2015)

32. H.B. Callen, Thermodynamics: An Introduction to the Physical Theories of the Equilibrium Thermostatics and Irreversible Thermodynamics (Wiley, New York, 1960)

33. K.A. Wiberg, Tetrahedron 24, 1083 (1968)

34. M.S. Gopinathan, K. Jug, Theor. Chim. Acta (Berl.) 63, 497, 511 (1983)

35. K. Jug, M. S. Gopinathan, in Theoretical Models of Chemical Bonding, vol. II, ed. by Z.B. Maksić, (Springer, Heidelberg, 1990), p. 77

36. I. Mayer, Chem. Phys. Lett. 97, 270 (1983)

37. R.F. Nalewajski, A.M. Köster, K. Jug, Theoret. Chim. Acta (Berl.) 85, 463 (1993)

38. R.F. Nalewajski, J. Mrozek, Int. J. Quantum Chem. 51, 187 (1994)

39. R.F. Nalewajski, S.J. Formosinho, A.J.C. Varandas, J. Mrozek, Int. J. Quantum Chem. 52, 1153 (1994)

40. R.F. Nalewajski, J. Mrozek, G. Mazur, Can. J. Chem. 100, 1121 (1996)

41. R.F. Nalewajski, J. Mrozek, A. Michalak, Int. J. Quantum Chem. 61, 589 (1997)

42. J. Mrozek, R.F. Nalewajski, A. Michalak, Polish J. Chem. 72, 1779 (1998)

43. R.F. Nalewajski, Chem. Phys. Lett. 386, 265 (2004)

44. R.F. Nalewajski, Mol. Phys. 104, 493 (2006)

45. J.E. Harriman, Phys. Rev. A 24, 680 (1981)

46. G. Zumbach, K. Maschke, Phys. Rev. A 28, 544 (1983); Erratum. Phys. Rev. A 29, 1585 (1984)

47. P. Hohenberg, W. Kohn, Phys. Rev. 136B, 864 (1964) 
48. W. Kohn, L.J. Sham, Phys. Rev. 140A, 1133 (1965)

49. M. Levy, Proc. Natl. Acad. Sci. USA 76, 6062 (1979)

50. W. Macke, Ann. Phys. (Leipzig) 17, 1 (1955)

51. T.L. Gilbert, Phys. Rev. B 12, 2111 (1975)

52. C.F. von Weizsäcker, Z. Phys. 96, 431 (1935) 